\title{
Respiratory and Telencephalic Modulation of Vocal Motor Neurons in the Zebra Finch
}

\author{
Christopher B. Sturdy, ${ }^{1}$ J. Martin Wild, ${ }^{2}$ and Richard Mooney ${ }^{1}$ \\ ${ }^{1}$ Department of Neurobiology, Duke University Medical Center, Durham, North Carolina 27710, and 2Division of Anatomy, Faculty of Medical and Health \\ Sciences, University of Auckland, Auckland 92019, New Zealand
}

Birdsong, like speech, involves coordinated vocal and respiratory activity achieved under telencephalic control. The avian vocal organ, or syrinx, is innervated by motor neurons (MNs) in the tracheosyringeal part of the hypoglossal nucleus (XIIts) that receive their synaptic input from medullary respiratory areas and telencephalic song control areas. Despite the importance of XIIts MNs to learned vocalizations, little is known about their intrinsic electrical properties or their synaptic inputs. Therefore, we made in vitro and in vivo intracellular recordings from XIIts MNs in adult male zebra finches to characterize their intrinsic properties and their synaptic modulation by respiratory and telencephalic areas. In vitro, electrical stimulation of ipsilateral or contralateral medullary respiratory areas (RAm) routinely evoked glycine receptor-mediated inhibition in XIIts. With inhibition blocked, similar stimulation evoked excitatory synaptic responses capable of driving sustained MN firing that was mediated partly by NMDA receptors. These inhibitory and excitatory inputs likely arise from RAm neurons, because chemical or electrical stimulation of RAm evoked similar responses in XIIts. In vivo, XIIts MNs displayed rhythmical, expiratory-related activity. EPSPs were pronounced at expiratory onset, but IPSPs were not apparent during inspiration, although XIIts MN firing was suppressed. However, hyperpolarizations as well as excitation were evoked by playback of the bird's own song, a stimulus that potently excites the telencephalic song nucleus that innervates XIIts. These findings illuminate functional properties of the songbird's brainstem circuitry and its specific activation by telencephalic inputs, which could coordinate vocal and respiratory activity during singing.

Key words: XIIts; RAm; birdsong; vocal learning; respiration; RA

\section{Introduction}

Oscine songbirds are one of the few nonhuman taxa to display vocal learning. Song learning, like speech learning, entails auditory-vocal integration and respiratory-vocal coordination under the control of the telencephalon (Doupe and Kuhl, 1999). Surprisingly little is known about synaptic mechanisms underlying these functions, although the songbird affords an excellent opportunity for their elucidation.

Birdsong is controlled by a set of interconnected brain nuclei that has as one of its final outputs the tracheosyringeal part of the hypoglossal motor nucleus (XIIts) and its efferent nerve, which innervates the syrinx, the avian vocal organ located at the confluence of the primary bronchi and trachea (Wild, 1997) (see Fig. 1). Because the XIIts motoneurons (MNs) do not innervate the tongue but rather muscles that are specifically involved in phonation and respiration and not articulation, they are more analogous to mammalian laryngeal than to lingual motoneurons (Wild and Zeigler, 1980; Vicario and Nottebohm, 1988; Dubbeldam and Bout, 1990; Vicario, 1991a; Goller and Suthers, 1996a,b). However, because the syrinx and larynx are separate structures in birds, XIIts MNs are not involved in those other behaviors that can engage the mammalian larynx, such as swallowing (Shiba et al., 1996). This apparent specialization of XIIts

\footnotetext{
Received July 19, 2002; revised Nov. 15, 2002; accepted Nov. 15, 2002.

This work was supported by National Institutes of Health Grant R01 DC 04691 to R.M. and J.M.W. and a National Sciences and Engineering Research Council of Canada Postdoctoral Fellowship to C.B.S.

Correspondence should be addressed to Richard Mooney, Department of Neurobiology, Box 3209, Duke University Medical Center, Durham, NC 27710. E-mail: mooney@neuro.duke.edu.

Copyright $\odot 2003$ Society for Neuroscience $\quad$ 0270-6474/03/231072-15\$15.00/0
}

for vocalization may simplify the analysis of XIIts MN synaptic connectivity. XIIts MNs receive synapses from several sources, including lateral medullary areas containing respiratory premotor neurons and the nucleus robustus archistriatalis (RA), a telencephalic structure essential to birdsong (Nottebohm et al., 1976; Wild, 1993a,b). Although the anatomy of these synaptic projections to XIIts is well described, little is known about their function.

In quiescent zebra finches (Taeniopygia guttata), XIIts MNs fire in phase with expiration. This respiratory rhythm is thought to be driven from lateral medullary regions projecting onto XIIts and containing respiratory premotor neurons (Manogue and Nottebohm, 1982; Williams and Nottebohm, 1985; Wild, 1993a; Wild et al., 2000). Still unresolved is whether the XIIts rhythm reflects expiratory-linked excitation or inspiratory-linked inhibition, or both. Clarification of this issue can provide information about how telencephalic and respiratory areas interact to effect vocalization.

During singing, syringeal and respiratory activity are modulated and coordinated (Suthers, 2000), presumably by RA, but the underlying synaptic mechanisms remain unknown. Middle and ventral RA neurons project topographically onto XIIts MNs that innervate either dorsal or ventral syringeal muscles, whereas dorsal RA neurons innervate respiratory premotor areas in the lateral medulla, among other targets (Vicario, 1991b; Vicario, 1993; Wild, 1993b; Reinke and Wild, 1998). Furthermore, respiration gates XIIts activity evoked by electrical stimulation of RA or its premotor afferent, $\mathrm{HVc}$ (used as a proper name) (Manogue and Nottebohm, 1982). Here we used in vitro and in vivo intra- 
cellular recordings from XIIts MNs to characterize their synaptic inputs from medullary respiratory areas. Then, we used song playback to assess telencephalic modulation of XIIts and respiratory activity in vivo. These experiments reveal functional features of XIIts MNs and associated brainstem circuits that could coordinate vocal and respiratory activity during singing.

\section{Materials and Methods}

Subjects. Eighty-five adult ( $>120$ post-hatch d) male zebra finches (Taeniopygia guttata) (23 for in vivo experiments, 62 for in vitro experiments) were used for the experiments, in accordance with a protocol approved by the Duke University Institutional Animal Care and Use Committee.

Song stimuli. Before the experiment, songs were recorded from a male zebra finch placed with an adult female zebra finch in a recording chamber (Industrial Acoustics, Bronx, NY). Songs were amplified and lowpass filtered at $10 \mathrm{kHz}$, digitized at $20 \mathrm{kHz}$ (National Instruments data acquisition board AT-MIO-16E2, Austin, TX), and stored on a hard drive. Songs were recorded and edited with LabView software (National Instruments; all custom software for this study was written by M. Rosen, F. Livingston, R. Neumann, and R. Balu, Duke University). Edited songs included one to six motifs and were $\sim 1-6 \mathrm{sec}$ in duration (a motif is the largest repeated unit in the bird's song and usually comprises single and multinote syllables; zebra finch song bouts typically consist of several motifs). Stimulus ensembles always included the bird's own song (BOS) and reversed BOS (i.e., song played backward). Forward and reverse versions of the BOS contain the same spectral energy, but differ in both their local and global temporal organization. Previous studies showed that RA projection neurons are potently excited by forward and not reverse BOS playback when the bird is under urethane (Doupe and Konishi, 1991).

In vivo preparatory surgery. On the morning of the day of the electrophysiological recording, birds were injected in the pectoral muscle with $20 \%$ urethane ( $90 \mu \mathrm{l}$ total; Sigma, St. Louis, MO), administered in $30 \mu \mathrm{l}$ doses at $30 \mathrm{~min}$ intervals. Approximately $1 \mathrm{hr}$ after the final injection, birds were anesthetized more deeply via inhalation of halothane gas (Halocarbon Laboratories, River Edge, NJ) and placed in a stereotaxic device (H. Adams, Caltech). After topical application of xylocaine $(2.5 \%)$ to the scalp and abdomen, the scalp was incised along the midline and a stainless steel post was mounted to the rostral part of the bird's skull with dental cement and super glue, and a pair of Teflon-coated platinumiridium wires ( 0.007 inch diameter) was inserted into the abdominal muscles to obtain expiratory electromyograms (EMGs). The bird was transferred to a sound-attenuating chamber (Industrial Acoustics) mounted on an air table (TMC, Peabody, MA), and the head was fixed in a metal frame at $45^{\circ}$ to the horizontal (Stokes et al., 1974) using the head post. A small craniotomy $(<300 \mu \mathrm{m}$ wide) was made over cerebellar folia corresponding to the location of the underlying XIIts nucleus, and the dura was torn with a fine insect pin (Minuten, Carolina Biological Supply). The bird was warmed with an electric blanket $\left(37^{\circ} \mathrm{C}\right.$; Harvard Apparatus, Holliston, MA).

In vivo electrophysiology and song presentation. Sharp electrodes (borosilicate glass, BF100-50-10; Sutter Instrument, Novato, CA) were pulled to yield a resistance of $100-200 \mathrm{M} \Omega$ when filled with $2 \mathrm{M}$ K-acetate and 5\% neurobiotin. A hydraulic microdrive (Soma Scientific, Irvine, CA) was used to lower electrodes into the nucleus ( $\sim 4 \mathrm{~mm}$ depth). Brief $(\sim 1 \mathrm{msec})$ capacitance overcompensation was used to "ring" the electrode to achieve entry into the cell. An AxoClamp 2B intracellular amplifier (Axon Instruments, Foster City, CA) was used in bridge mode to record intracellular membrane potentials, which were low-pass filtered at $3 \mathrm{kHz}$, digitized at $10 \mathrm{kHz}$, and stored on a PC hard drive. XIIts neurons were identified on-line by their action potential discharge properties in response to injected currents (see Results) and by the propensity to generate trains of action potentials in phase with expiration; in some cases, recording sites were verified histologically after the recording session. Spontaneous membrane potential and EMG recordings, 3-5 min long, were collected to inspect for respiratory-linked action potential discharge in XIIts MNs. Generally, 10-30 iterations of each auditory stimulus, delivered at intervals ranging from 6 to $10 \mathrm{sec}$, were presented at $\sim 70 \mathrm{~dB}$ (rms, A-weighting) through a speaker positioned $20 \mathrm{~cm}$ directly in front of the bird, using custom LabView software. Peristimulus time histograms (PSTHs; 25 msec bin width) and median-filtered averaged membrane potentials were computed off-line (see Data analysis).

When possible, the intrinsic properties of an impaled cell were also studied, after characterization of the respiratory rhythm of the cell and its auditory responses to BOS playback. Hyperpolarizing responses to negative current pulses $(-200$ to $-400 \mathrm{pA}, 1 \mathrm{sec}$ duration, measured at steady state) were collected to estimate input resistance, and instantaneous and mean firing rates, as well as the latency to the first spike, were calculated in response to positive current pulses $(+200$ to $+1000 \mathrm{pA}, 1$ $\mathrm{sec}$ duration). Cells were then filled with neurobiotin using positive currents $(+0.5$ to $+1 \mathrm{nA}, 500 \mathrm{msec}$ at $1 \mathrm{~Hz})$.

EMG signals were amplified $(10,000 \times)$ and band-pass filtered $(0.3-3$ $\mathrm{kHz}$ ) by an A-M Systems differential AC amplifier (A-M Systems, Everett, WA; model 1700). The signals were then digitized at $10 \mathrm{kHz}$ and stored on a PC hard drive. A BOS playback was considered "inspirationlinked" if it began during inspiration or within $200 \mathrm{msec}$ after the start of an inspiratory epoch, as determined by visually inspecting the expiratory EMG (i.e., for periods of expiratory EMG inactivity). A BOS playback was considered to be "expiration linked" if it began during expiration or within $200 \mathrm{msec}$ after the onset of expiration, as determined by visually inspecting the expiratory EMG (i.e., for expiratory EMG activity).

$H V c$ inactivation. To confirm that XIIts MNs received synaptic drive from telencephalic song nuclei, we recorded XIIts activity extracellularly in two urethane-anesthetized birds before, during, and after concentrated GABA [0.25 $\mathrm{m}$ in artificial CSF (ACSF)] infusion into HVc. A glass recording micropipette (tip outer diameter 10-15 $\mu \mathrm{m}$ ) filled with $1 \mathrm{M}$ $\mathrm{NaCl}$ was lowered through the caudal cerebellum into XIIts, as identified by its depth from the cerebellar surface $(\sim 4.4 \mathrm{~mm})$, its distance from the midline $(0.4 \mathrm{~mm})$, and the presence of a marked respiratory rhythm. Multiunit recordings were then obtained while forward and reverse BOS were presented as detailed above. After detection of BOS-evoked activity in XIIts, concentrated GABA was infused into the ipsilateral $\mathrm{HVc}$ using a Picospritzer (General Valve, Fairfield, NJ; each pulse was $25 \mathrm{msec}$ duration at $25 \mathrm{psi}$ ), starting with 10 pulses before song playback and then 2 pulses during each interstimulus interval, sustained for a total of 5-15 stimulus iterations; XIIts activity was monitored through the period of GABA application and until forward BOS-evoked responses again became evident $(\sim 2-5 \mathrm{~min})$. Similar size applications have been used to inactivate HVc in other studies conducted in this lab (R. Mooney, personal observation).

Retrograde labeling of XIIts MNs. To verify that the neurons that we recorded in the slice were syringeal and not lingual MNs [which lie rostroventral to XIIts MNs (Wild, 1993b)], we retrogradely labeled XIIts MNs before in vitro recording sessions in a subset of birds by injecting ventral syringeal muscles with 5\% biotinylated dextran amine (BDA) (Molecular Probes, Eugene, OR; dissolved in 0.025 м PBS) (Vicario and Nottebohm, 1988). With the bird under equithesin anesthesia $(2 \mathrm{mg} / \mathrm{kg}$, i.m.; 0.85 gm chloral hydrate, $0.21 \mathrm{gm}$ pentobarbitol, $0.42 \mathrm{gm} \mathrm{MgSO}_{4}, 2.2$ $\mathrm{ml} 100 \%$ ethanol, $8.6 \mathrm{ml}$ propylene glycol brought to $20 \mathrm{ml}$ final volume with $\mathrm{dH}_{2} \mathrm{O}$ ), a midline incision was made in the upper thorax to expose the interclavicular air sac, which was then ruptured to reveal the syrinx. The ventral syringeal muscles were then pressure injected bilaterally with $\sim 5 \mu \mathrm{l}$ of BDA via a glass micropipette attached to a Picospritzer, under visual guidance. The incision site was then sutured and coated with Neosporin. Birds were allowed to recover for transport of the BDA for 2-14 d before they were used in an experiment.

Brain slices. These experiments used electrophysiological techniques that have been described extensively in previously published studies (Mooney, 1992; Livingston and Mooney, 1997). Briefly, transverse brain slices from the medulla of adult (age 123-469 post-hatch d) male zebra finches were cut at $400 \mu \mathrm{m}$ and transferred to a holding chamber (room temperature). Intracellular recordings were made using an interface-type chamber $\left(30^{\circ} \mathrm{C}\right.$; Medical Systems). ACSF consisted of (in mM): $119 \mathrm{NaCl}$, $2.5 \mathrm{KCl}, 1.3 \mathrm{MgCl}_{2}, 2.5 \mathrm{CaCl}_{2}, 1 \mathrm{NaH}_{2} \mathrm{PO}_{4}, 26.2 \mathrm{NaHCO}_{3}, 11$ glucose, equilibrated with $95 \% \mathrm{O}_{2} / 5 \% \mathrm{CO}_{2}$. Equiosmolar sucrose was substituted for $\mathrm{NaCl}$ during slicing.

In vitro electrophysiology. Sharp electrodes (borosilicate glass, BF100- 
50-10, Sutter Instrument) were pulled to yield a resistance of 100-200 $\mathrm{M} \Omega$ when filled with $2 \mathrm{M} \mathrm{K}$-acetate and $5 \%$ neurobiotin. A motorized microdrive (Newport Scientific, Irvine, CA; model 860A) was used to lower electrodes into the nucleus, which was visible under epiillumination as a circular structure situated $\sim 0.5 \mathrm{~mm}$ from the midline. Brief $(\sim 1 \mathrm{msec})$ capacitance overcompensation was used to ring the electrode to achieve entry into the cell. An AxoClamp 2B intracellular amplifier (Axon Instruments) was used in bridge mode to record intracellular membrane potentials, which were low-pass filtered at $3 \mathrm{kHz}$, digitized at $10 \mathrm{kHz}$, and stored on a PC hard drive. Only cells with resting potentials negative of $-50 \mathrm{mV}$ and overshooting spikes were used for analysis.

Hyperpolarizing responses to negative current pulses $(-200$ to -600 $\mathrm{pA}$, measured during steady state) were collected to estimate input resistance, and instantaneous and mean firing rates were calculated in response to positive current pulses ( $+200-1000 \mathrm{pA}, 1 \mathrm{sec}$ duration). Concentric bipolar stimulating electrodes (Frederic Haer, Bowdoinham, $\mathrm{ME}$ ) were placed in the lateral medulla, and synaptic potentials in XIIts were evoked using brief $(100 \mu \mathrm{sec})$ currents of $50-600 \mu \mathrm{A}$. In brain slices of the closed medulla, which often included the obvious commissura infima dorsal to the central canal, these electrodes were located in an area corresponding to the location of the lateral part of nucleus retroambigualis (RAm) (Wild, 1993a), which was discernible under epiillumination as an arc extending ventrolaterally from XIIts. In slices that included the obex region, however, it was not possible to determine whether the electrodes were located only in RAm because of the additional presence at these levels of nucleus parambigualis (PAm), the source of inspiratory-related bulbospinal neurons that is rostrally continuous with RAm and also projects onto XIIts (Reinke and Wild, 1998). The stimulating electrodes were moved dorsal or ventral to RAm to confirm the specificity of the stimulation locus from which the response was evoked. To verify that the effects of electrical stimulation were caused by the activation of cell bodies and not of fibers of passage, $10 \mathrm{~mm}$ glutamate was applied to the site of effective stimulation via a puffer pipette, using brief ( $5 \mathrm{msec}$ at $15 \mathrm{psi}$ ) pulses supplied by a Picospritzer. Once data collection was completed, cells were filled with neurobiotin using positive currents $(+0.5$ to $+1 \mathrm{nA}, 500 \mathrm{msec}$ at $1 \mathrm{~Hz})$.

In vivo data analysis. The suprathreshold responsiveness $\left(R_{\text {supra }}\right)$ of cells with spiking activity was calculated by $R_{\text {supra }}=S_{\mathrm{FR}}-B_{\mathrm{FR}}$, where $S_{\mathrm{FR}}$ and $B_{\mathrm{FR}}$ are the firing rates during each stimulus presentation and during a $2.0 \mathrm{sec}$ baseline period before each stimulus presentation, respectively. To assess subthreshold responsiveness in spiking and nonspiking cells, raw traces were first median filtered (each point was replaced by the median value of the surrounding 50 points, equivalent to $5 \mathrm{msec}$ at the 10 $\mathrm{kHz}$ sampling rate used here). Median filtering removed the action potential, which was typically $\sim 1 \mathrm{msec}$ in duration, yet did not distort slower membrane potential movements (Jagadeesh et al., 1997, their Fig. $1)$. The subthreshold depolarizing responsiveness $\left(R_{\mathrm{Vm}}\right)$ of these cells was measured by $R_{\mathrm{Vm}}=S_{\text {area }}-B_{\text {area }}$, where $S_{\text {area }}$ and $B_{\text {area }}$ are the integrals of the positive-going deviations in membrane potential $\left(V_{\mathrm{m}}\right)$ either during (i.e., $S_{\text {area }}$ ) or before (i.e., $B_{\text {area }}$ ) stimulus presentation relative to the mode membrane potential measured during the baseline period. That is, the total positive area during the baseline (measured from the mode) was subtracted from the total positive area during the stimulus (measured from the mode) [for examples of how these subthreshold values were measured, see Mooney (2000), his Fig. 9]. The mode membrane potential was calculated for the baseline data array with an automated LabView routine and was used instead of the mean or median of the baseline data array because we observed that it gave the most reliable measure of the central tendency of the baseline membrane potential. Similar calculations were made for the subthreshold hyperpolarizing area, but instead using the negative-going deviations in membrane potential from the baseline mode value. The net subthreshold hyperpolarization was multiplied by -1 , so that a net increase in subthreshold hyperpolarization relative to the baseline would be represented by a negative number. It was possible for single cells to respond to a stimulus with an increase in both negative and positive area relative to baseline. Furthermore, strong hyperpolarizing responses to a stimulus could yield negative values for positive and negative area (e.g., when the baseline positive area was greater than positive area evoked by the stimulus). Average $R_{\text {supra }}$ or $R_{\mathrm{Vm}}$ values were computed for 10-40 stimulus iterations. Significance was determined with paired $t$ tests comparing stimulus-evoked suprathreshold, subthreshold depolarizing, or subthreshold hyperpolarizing responses to corresponding baseline measures.

To compare suprathreshold and subthreshold responses, response strengths were expressed as $z$-scores. The suprathreshold $z$-score $\left(Z_{\text {supra }}\right)$ is the difference between the average firing rate during the stimulus and during a $1.5 \mathrm{sec}$ baseline period before stimulus presentation, divided by the SD of this difference:

$$
Z_{\text {supra }}=\frac{\bar{S}_{\mathrm{FR}}-\bar{B}_{\mathrm{FR}}}{\sqrt{\operatorname{Var}\left(S_{\mathrm{FR}}\right)+\operatorname{Var}\left(B_{\mathrm{FR}}\right)-2 \operatorname{Covar}\left(S_{\mathrm{FR}}, B_{\mathrm{FR}}\right)}},
$$

where ${ }^{-} S_{\mathrm{FR}}$ is the mean firing rate during the stimulus and ${ }^{-} B_{\mathrm{FR}}$ is the mean firing rate during the baseline period, and the denominator is the $\mathrm{SD}$ of $S_{\mathrm{FR}}-B_{\mathrm{FR}}$. For nonspiking cells and median-filtered spiking cells, the subthreshold $z$-score $\left(Z_{\mathrm{Vm}}\right)$ is given by the difference between the average area during stimulus presentation and that during baseline, divided by the $\mathrm{SD}$ of this difference. The $Z_{\mathrm{Vm}}$ formula is the same as that for $Z_{\text {supra }}$, with substitutions of area for FR, where ${ }^{-} S_{\text {area }}$ is the mean deviation in $V_{\mathrm{m}}$ (from the baseline mode, calculated separately for negative or positive area, as stated above) during song presentation, and ${ }^{-} B_{\text {area }}$ is the mean deviation in $V_{\mathrm{m}}$ during baseline; the denominator is the SD of $S_{\text {area }}$ $-B_{\text {area }}$.

The selectivity of a neuron for forward over reverse BOS playback was measured using the psychophysical metric $d^{\prime}$ (Green and Swets, 1966):

$$
d_{\text {supra }}^{\prime}=\frac{2\left(\bar{R}_{\mathrm{FR}_{\mathrm{BOS}}}-\bar{R}_{\mathrm{FR}_{\mathrm{rev}}}\right)}{\sqrt{\sigma_{\mathrm{BOS}}^{2}+\sigma_{\mathrm{rev}}^{2}}}
$$

or

$$
d_{\mathrm{V}_{\mathrm{m}}}^{\prime}=\frac{2\left(\bar{R}_{\text {areaвos }}-\bar{R}_{\text {area }}\right)}{\sqrt{\sigma_{\text {BOS }}^{2}+\sigma_{\text {rev }}^{2}}},
$$

which estimates the discriminability between two stimuli. A difference in response to these two stimuli has been used previously as the criterion for auditory selectivity of neurons in $\mathrm{HVc}$, as well as in other song nuclei (Solis and Doupe, 1997; Theunissen and Doupe, 1998; Janata and Margoliash, 1999; Mooney, 2000; Rosen and Mooney, 2000). The $d^{\prime}$ value comparing the response to BOS relative to reversed BOS is given by $d^{\prime}{ }_{\text {supra }}$, which represents suprathreshold responsiveness, and $d^{\prime}{ }_{V m}$ represents subthreshold responsiveness. ${ }^{-} R$ is the mean value of $R$ (as described above), and $\sigma^{2}$ is its variance. This selectivity measure resembles a ratio measure but accounts for both the mean and the variance of the responses of a cell and can report negative values. A $d^{\prime}$ value $>0.7$ (or $<$ -0.7 , reflecting an excitatory bias toward reverse BOS) was the criterion used for terming a cell "selective"; this corresponded to $p=0.036$ as measured by a paired $t$ test comparing $R_{\mathrm{Vm}}$ or $R_{\mathrm{FR}}$ values with 20 presentations of BOS and reversed BOS. Note that $d^{\prime}$ values for subthreshold responses $\left(d_{V m}^{\prime}\right)$ were calculated separately for positive and negative areas. Tests for statistical significance are reported in the figure legends, except as noted in Results.

In vivo and in vitro intrinsic data analysis. A software threshold event detector was used to measure instantaneous spike rates throughout the 1 sec period of positive current injection. Action potential half-height widths were measured from the shoulder of the spike, where the membrane potential described a sharp positive inflection. The spike afterhyperpolarization (AHP) was measured from the spike shoulder to the post-spike hyperpolarization trough. Similarly, afterdepolarizations (ADPs) were measured from the trough of the spike AHP to the peak of the spike ADP. Resting potential was determined by subtracting any DC offset observed after electrode withdrawal. Input resistance measurements were calculated by measuring the steady-state voltage caused by injecting small $(-200 \mathrm{pA})$ hyperpolarizing current pulses. All values reported are mean \pm SEM; statistics and tests for significance are in Results. 
In vivo and in vitro histology. After an in vivo recording session the bird was deeply anesthetized with equithesin and transcardially perfused with $0.9 \%$ saline for $3 \mathrm{~min}$, followed by $4 \%$ paraformaldehyde (PFA) in $25 \mathrm{~mm}$ sodium phosphate buffer for $30 \mathrm{~min}$. The brain was removed and postfixed in $4 \%$ PFA with $30 \%$ sucrose overnight, blocked coronally, and sectioned on a freezing microtome at $60 \mu \mathrm{m}$. Brain slices were immersion fixed in $4 \%$ PFA in $25 \mathrm{~mm}$ sodium phosphate buffer and equilibrated in $30 \%$ sucrose overnight before sectioning on a freezing microtome at 60 $\mu \mathrm{m}$. Both whole brain and brain slice sections were either processed using standard HRP-DAB reaction techniques (Kittelberger and Mooney, 1999) or visualized using Alexafluor 488 (Molecular Probes); sections were incubated overnight at $4^{\circ} \mathrm{C}$ in a 1:700 dilution of Alexafluor 488 and $0.4 \%$ Triton X-100 in 0.025 M PBS, rinsed in PBS three times for 10 min each time, mounted, and coverslipped.

\section{Results}

To directly assess the function of synaptic inputs that XIIts MNs receive from respiratory areas in the lateral medulla, we made transverse brain slices through the level of the syringeal portion of the hypoglossal motor nucleus in adult male zebra finches (Fig. 1). We first characterized the intrinsic properties of morphologically identified XIIts MNs during in vitro recording sessions and then used electrical and chemical stimulation techniques to activate synaptic inputs to XIIts from respiratory areas in the lateral medulla. We then made in vivo intracellular recordings from XIIts MNs to measure synaptic activity during normal respiration and when telencephalic inputs to XIIts were activated in a naturalistic manner by song playback.

\section{XIIts MN properties measured in vitro}

In transverse slices through the caudal medulla, the hypoglossal nucleus is a distinct, round structure close to the central canal. But because the tracheosyringeal (XIIts) and lingual (XIIl) parts of the hypoglossal nucleus slightly overlap rostral to the obex, we conducted initial experiments to confirm that we were recording from XIIts and not XIIl MNs. We first labeled tracheosyringeal MNs by injecting retrograde tracers into the ventral syringeal muscles and then we intracellularly stained individual XIIts MNs in the slice. As expected of XIIts MNs, the impaled cells were within the field of retrogradely labeled cell bodies (Fig. $2 \mathrm{~A}$ ), with extensive dendrites and unbranched axons exiting the nucleus ventrally to enter the XIIts nerve root. Thereafter, we recorded from the part of the hypoglossal nucleus caudal to the obex, which contains only XIIts and not XIIl MNs (Wild, 1993a).

The XIIts neurons that we recorded from displayed remarkable homogeneity in their intrinsic properties. In vitro, XIIts MNs had average resting potentials of $-64 \pm 1 \mathrm{mV}$ (mean \pm SEM) and average input resistances of $49 \pm 3 \mathrm{M} \Omega$ in vitro (Table 1 ). Although XIIts MNs recorded in vitro showed no evidence of rhythmic action potential discharge like the rhythmic respiratory activity that they display in vivo (see below), a subset of cells recorded in vitro exhibited tonic action potential discharge (22 of 40 cells; $\sim 24 \mathrm{~Hz}$ ). Positive currents passed through the recording electrode-elicited action potential trains from XIIts MNs (Fig. $2 B$ ), the mean discharge rates of which increased in a linear manner with injected positive currents, up to an amplitude of $+1 \mathrm{nA}$ in vitro (Fig. $2 D$ ). The instantaneous frequency of these evoked action potential trains dropped over the first few interspike intervals and then remained fairly constant over the remainder of the injected pulse (Fig. 2B,E). Spike AHPs and ADPs (noted as a positive peak immediately after spike) were observed in all cells (40 of 40 cells), and anomalous rectification, evinced as a sag in the hyperpolarizing response to a negative current pulse, was seen in a subset of cells (14 of 40 cells) (Table 1).

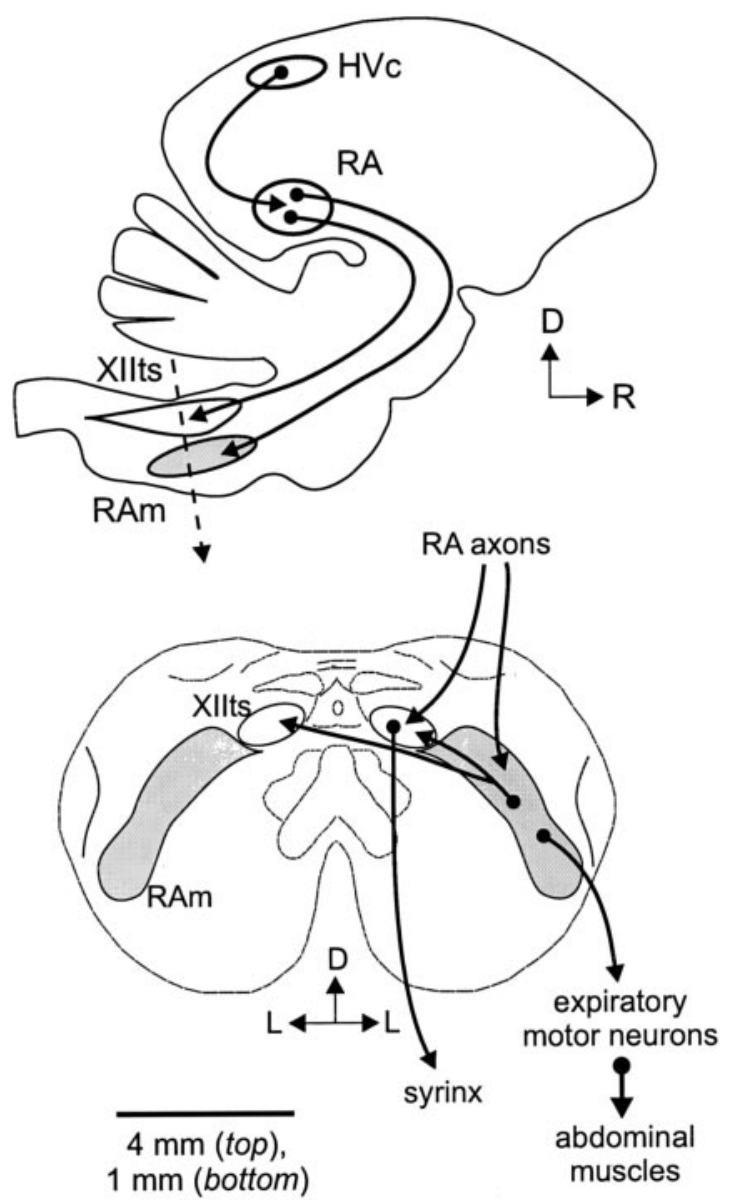

Figure 1. These simplified diagrams emphasize the major vocal and respiratory pathways important for the production of learned vocalizations in the male zebra finch. At top is a sagittal view of the descending axons from the telencephalic song nucleus robustus archistriatalis $(R A)$ to vocal and respiratory areas in the brainstem. At the bottom is a transverse brainstem section (made at the level of the dashed arrow in the sagittal view) resembling the orientation of the brain slices that we recorded from and showing the tracheosyringeal part of the hypoglossal nucleus (XIIts), which contains the motor neurons innervating the muscles of the avian song organ, or syrinx. XIIts receives ipsilateral and contralateral afferents from the nucleus retroambigualis (RAm), which also contains expiratory bulbospinal premotor neurons and is likely to be the source of expiratory-related activity detected in nXIlts and in the tracheosyringeal nerve. XIlts neurons also receive synaptic input from the nucleus parambigualis (data not shown), which contains inspiratory bulbospinal neurons, and from the ventrolateral parabrachial nucleus, the nucleus infra-olivaris superior, and the ventrolateral nucleus of the rostral medulla (data not shown) (Wild et al., 1990; Wild, 1993a, 1994). XIlts and RAm each receive afferents from the telencephalic song motor nucleus RA, as well as from the dorsomedial nucleus of the intercollicular region of the midbrain (data not shown) (Wild, 1993b; Wild et al., 1997). RA receives auditory and motor input from the song nucleus HVc (used here as a proper name); HVc and RA are essential to the production of learned vocalizations in songbirds. $D$, Dorsal; $R$, rostral; $L$, lateral.

\section{Synaptic inputs from lateral medullary respiratory centers to XIIts studied in vitro}

To probe directly the synaptic connections made by respiratory centers located in the lateral medulla onto XIIts MNs, we made intracellular recordings from XIIts MNs in brain slices and applied focal electrical stimulation to the region containing either the ipsilateral or contralateral RAm (Fig. 3 depicts a summary schematic of stimulation sites). Previous studies showed that RAm consists of a narrow band of cells extending laterally from XIIts, swelling slightly in extent as it approaches the lateral border of the medulla (Figs. 1, 3) (see also Wild, 1993a). In practice, we found that the stimulating electrode evoked synaptic responses in 

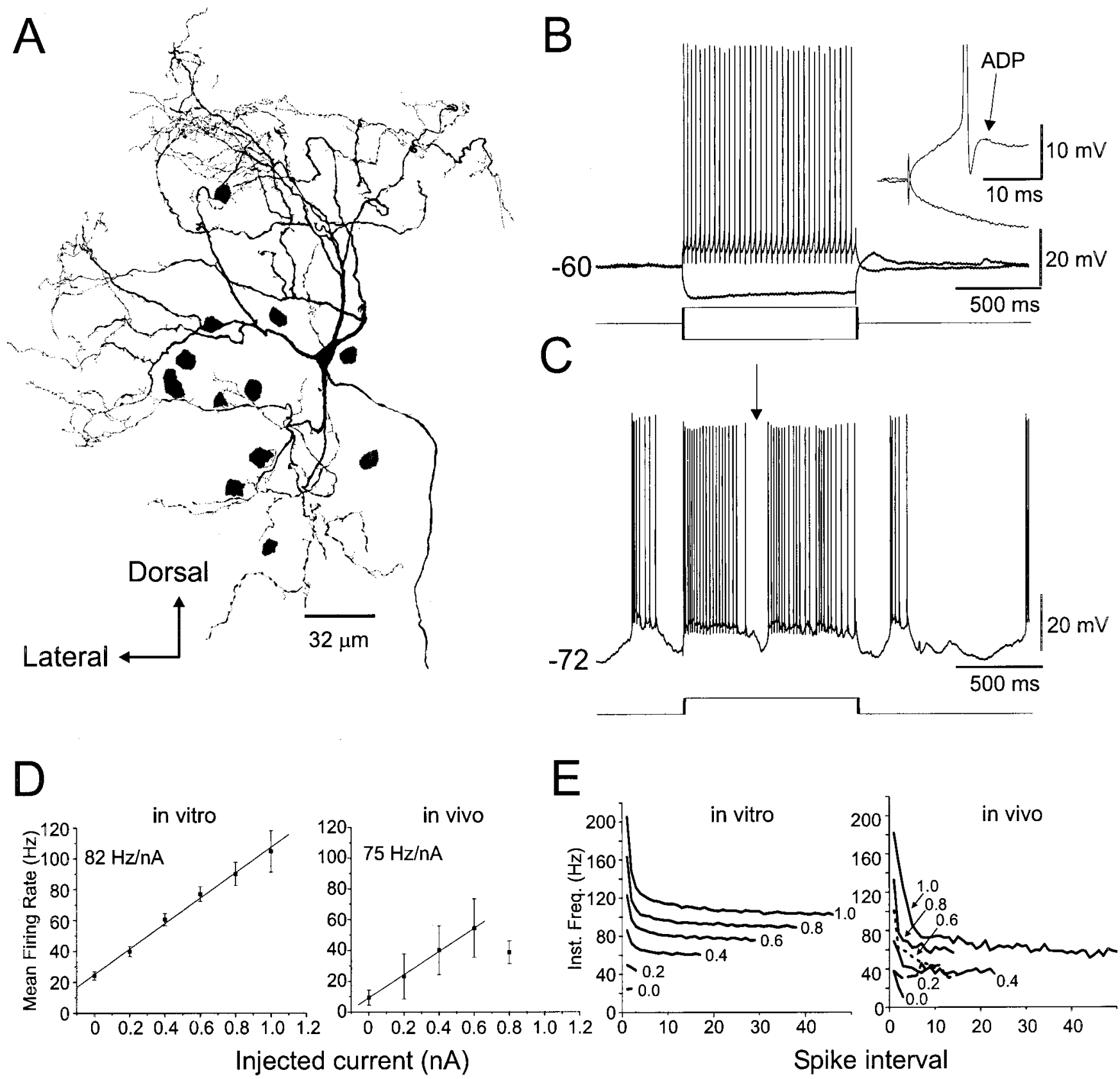

Figure 2. Morphological and intrinsic electrical features of XIIts MNs. A, Intracellular staining of an XIIts MN shows dendrites extending into regions surrounding $n X I I t s$ and an axon that travels ventrally along the midline toward the XIlth nerve rootlet. Motor neurons ( gray polygons) in the rostral portion of Xllts were labeled by injection of retrograde tracer into the ventral syringeal muscle several days before intracellular filling in a brain slice preparation. $D$, Dorsal; $L$, lateral. $B$, In vitro, Xllts MNs generated highly regular trains of action potentials in response to positive currents. Membrane potential responses of an XIlts MN to positive and negative injected currents ( $\pm 200 \mathrm{pA}$; the pulse duration is shown below the superimposed voltage traces) passed through the recording electrode are shown from an in vitro intracellular recording made in a brain slice. The inset trace highlights the spike afterdepolarization (ADP) characteristic of XIlts MNs. C, An in vivo intracellular membrane potential recorded from a Xllts MN shows that in response to a $+200 \mathrm{pA}$ current injected through the recording electrode, the cell generates a train of action potentials similar to those seen in vitro, but with action potential suppression, likely to be occurring during the inspiratory phase of respiration (arrow) (see Fig. 8 and Results). Note that subthreshold membrane potential movement during this inspiratory phase consists of a gradual rather than abrupt hyperpolarization. $D$, The average firing frequencies (in hertz; in vitro, left; in vivo, right) are plotted as a function of the amplitude of the positive current injected through the recording electrode for populations of XIlts MNs (in vitro, 40 cells; in vivo, 5 cells). The average firing frequency as a function of injected current in vitro and in vivo was highly linear between 0 and $+0.6 \mathrm{nA}$. E, Instantaneous action potential frequency plots (in vitro, left; in vivo, right; averages from same cells as in D).

XIIts from very restricted sites in the lateral medulla. These "hot spots" approximated the diameter of the stimulating electrode $(0.5 \mathrm{~mm})$ and were located in the region known to include RAm.

Ipsilateral stimulation of RAm evoked IPSPs in almost all XIIts MNs tested (Fig. 3A, top trace) (136 of 145 cells; 40 birds), whereas it evoked EPSPs in a small fraction of cells tested ( 9 of 145 cells; 40 birds) (Fig. 3A, bottom trace). In a smaller number of cells, we also examined whether the lateral medulla provided functional synaptic input to the contralateral XIIts. Indeed, contralateral RAm stimulation led to detectable PSPs in almost half the cells tested ( 8 of 18 cells; nine birds) (Fig. 3B); these consisted of both IPSPs ( 4 of 18 cells, three birds) and EPSPs ( 4 of 18 cells; three birds). Stimulation of lateral medullary sites either just dorsal or ventral to the area of RAm (i.e., movement of one diameter 


\begin{tabular}{|c|c|c|c|c|c|c|c|c|c|}
\hline & $V_{\text {rest }}(\mathrm{mV})$ & Input resistance $(\mathrm{M} \Omega$ ) & Tonic firing rate (Hz) & $\begin{array}{l}\text { Action potential } \\
\text { width half-height } \\
\text { (msec) }\end{array}$ & ADP/AHP & AHP amplitude (mV) & ADP amplitude (mV) & $\begin{array}{l}\text { Firing frequency } \\
(\mathrm{Hz} / \mathrm{nA})\end{array}$ & Rectification $(n)$ \\
\hline In vitro & $-63.8 \pm 1.3(22)^{a}$ & $49.4 \pm 3.3(33)$ & $\begin{array}{l}24.1 \pm 2.7(22) \\
\text { Expiratory-linked }\end{array}$ & $0.49 \pm 0.02(40)$ & $40(40)$ & $14 \pm 0.7$ (38) & $5.7 \pm 0.5(27)$ & $82(40)$ & $14(40)$ \\
\hline In vivo & $-60 \pm 3.8(6)$ & $49.2 \pm 10.8(5)$ & rhythmic firing & $0.68 \pm 0.1(6)$ & $6(6)$ & $7.8 \pm 1(5)$ & $3.6 \pm 0(2)$ & $75(5)$ & $3(6)$ \\
\hline
\end{tabular}

${ }^{a}$ Number of cells in parentheses.

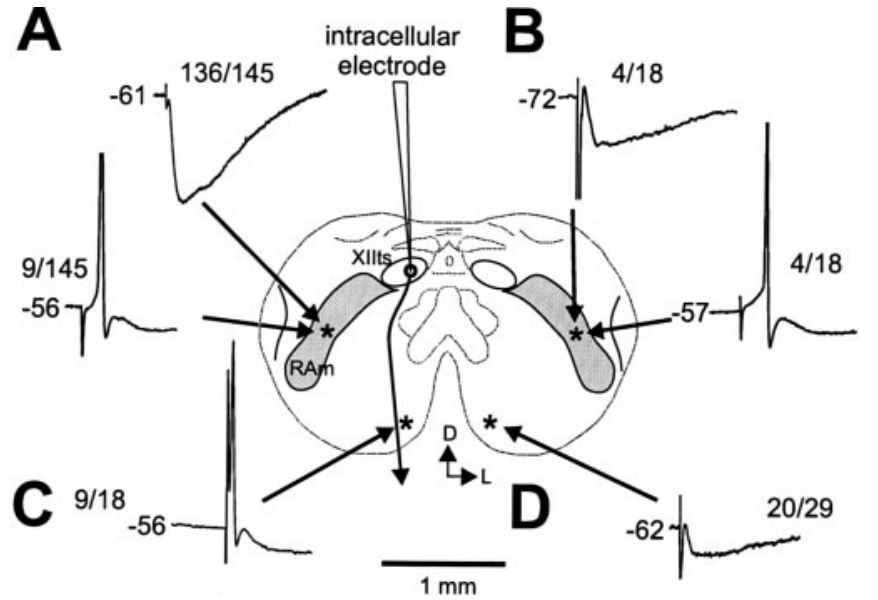

Figure 3. A schematic summarizing the types and frequencies of synaptic and antidromic responses evoked in XIIts MNs after electrical stimulation at different sites (marked by asterisks) within the transverse brain slice preparation of the caudal medulla. $A$, Stimulation in ipsilateral RAm predominantly evoked IPSPs and rarely evoked EPSPs. B, Contralateral RAm stimulation evoked IPSPs and EPSPs from a small fraction of the cells tested. C, D, Stimulation in the Xllts nerve root evoked antidromic spikes (ipsilateral stimulation) and IPSPs (contralateral stimulation) in XIlts MNs. The numbers in parentheses refer to the number of cells showing the response illustrated by the accompanying trace of the total number of cells tested. D, Dorsal; $L$, lateral.

of the stimulating electrode away from RAm) did not evoke PSPs in XIIts MNs.

Responses in XIIts MNs were also detected after electrical stimulation of both the ipsilateral and contralateral hypoglossal rootlets (Fig. 3C,D). Although direct stimulation of the XIIts nucleus itself was not attempted, it is probable that the stimulating electrode selectively activated ipsilateral XIIts MNs via antidromic propagation of the spike via the nerve root. Consistent with this idea, stimulation of the ipsilateral hypoglossal rootlet evoked antidromic spikes in half of the XIIts MNs that we tested (9 of 18 cells; three birds). Unexpectedly (see Discussion), stimulation of the ipsilateral and contralateral hypoglossal nerve root could also evoke synaptic responses in XIIts. Specifically, stimulation of the ipsilateral hypoglossal rootlet evoked IPSPs in 7 of 18 cells in three birds, whereas stimulation of the contralateral hypoglossal rootlet evoked IPSPs in a majority of cells tested (20 of 29 cells; four birds) and an EPSP in one cell (data not shown). In the case of responses evoked by contralateral nerve root stimulation, we assume that XIIts MNs ipsilateral to the stimulation site were synaptically interposed between the stimulation and recording sites, because XIIts axons project only ipsilaterally (Wild, 1997) (see Discussion).

\section{Synaptic pharmacology}

The mean reversal potential of IPSPs $(-87 \pm 6 \mathrm{mV} ; n=7$ cells from 5 birds) evoked in XIIts MNs by RAM stimulation is consistent with either glycine or GABA receptor mediated inhibition. To better characterize inhibitory synaptic inputs from the lateral

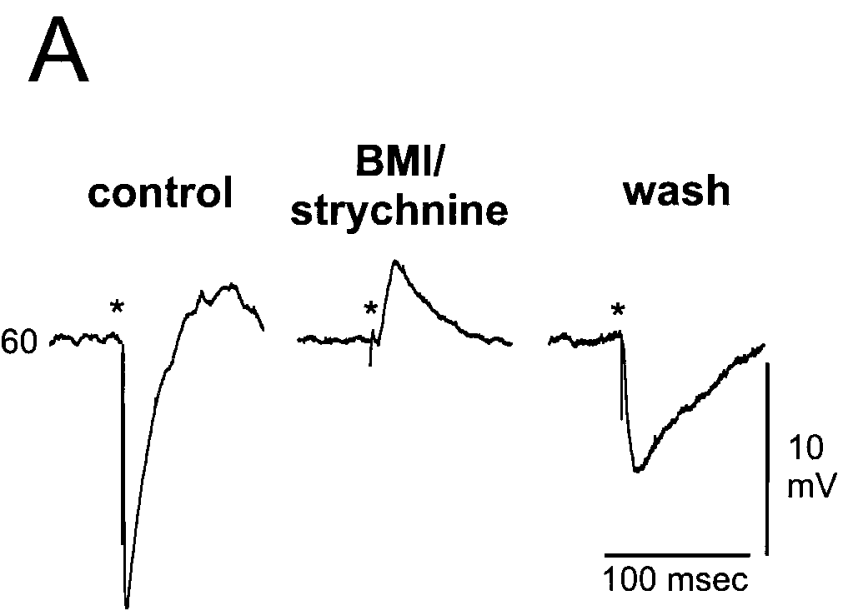

$B$

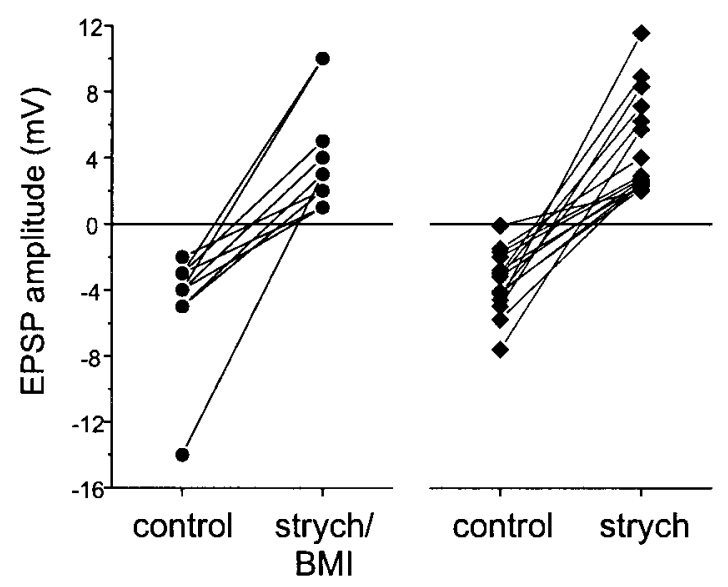

Figure 4. Electrical stimulation in RAm evokes synaptic inhibition in Xllts MNs mediated predominantly by glycine receptors. $A$, IPSPs evoked in XIlts MNs by ispilateral RAm stimulation (Fig. $3 A$ ) were abolished by combined application of $\mathrm{GABA}_{A}$ and glycine receptor blockers (BMI and strychnine; see Materials and Methods for details). This treatment reversibly abolished the IPSPs elicited in XIIts MNs and unmasked EPSPs. An asterisk marks stimulation time. B, Scatterplots of PSP amplitude before (control) and immediately after treatment with strychnine and BMI (left) or strychnine alone (right).

medulla onto XIIts MNs, we used a puffer pipette to apply focally GABA- and glycine-receptor blockers [100 $\mu \mathrm{M}$ bicuculline methiodide (BMI) and strychnine dissolved in ACSF] to the XIIts intracellular recording site while electrically stimulating synaptic inputs from the ipsilateral RAm. These drugs applied together abolished the IPSP in all cells that we tested (10 cells; eight birds), and in all such cases, rather than simply failing to evoke any response, electrical stimulation then elicited an EPSP (Fig. 4, Table 2) (mean IPSP amplitude, $-4.7 \pm 1 \mathrm{mV}$; mean EPSP amplitude, $\left.+4.1 \pm 1 \mathrm{mV} ; t_{(9)}=-6.26 ; p<.001\right)$. In an attempt to 
Table 2. Synaptic properties of XIIts MNs in vitro

\begin{tabular}{|c|c|c|c|c|c|c|c|}
\hline IPSP (control) & $\begin{array}{l}\text { PSP (strychnine } \\
\text { and BMI) }\end{array}$ & IPSP (control) & PSP (strychnine) & $\begin{array}{l}\text { "Late" portion of } \\
\text { EPSP (strychine) }\end{array}$ & $\begin{array}{l}\text { "Late" portion of } \\
\text { EPSP (strychine, } \\
\text { APV) }\end{array}$ & $\begin{array}{l}\text { "Late" portion of } \\
\text { EPSP (strychine) }\end{array}$ & $\begin{array}{l}\text { "Late" portion of } \\
\text { EPSP (strychine, } \\
-V_{m} \text { ) }\end{array}$ \\
\hline$-4.7 \pm 1.1(10)^{a}$ & $\begin{array}{l}+4.1 \pm 1(10) \\
\operatorname{Vm}(\mathrm{mV}) \\
\operatorname{Ri}(\mathrm{M} \Omega)\end{array}$ & $\begin{aligned}-3.5 & \pm 0.4(16) \\
-63.5 & \pm 1.5 \\
51.1 & \pm 7.1\end{aligned}$ & $\begin{aligned}+\mathbf{4} & \pm \mathbf{0} \pm \mathbf{0 . 7}(16) \\
-62.7 & \pm 1.2 \\
53.7 & \pm 6.2\end{aligned}$ & $\begin{array}{c}+5.2 \pm 0.8(8) \\
-65.9 \pm 2.7 \\
72.4 \pm 6\end{array}$ & $\begin{array}{c}+\mathbf{+ 3 . 5} \pm \mathbf{0 . 7}(8) \\
-64.9 \pm 1.3 \\
74 \pm 8.5\end{array}$ & $\begin{array}{c}+4.4 \pm 0.8(8) \\
63 \pm 1.8\end{array}$ & $\begin{array}{l}+2.9 \pm 0.6(8) \\
-82.9 \pm 3.7\end{array}$ \\
\hline
\end{tabular}

Bold indicates significant difference from relevant previous value. All PSP values are in millivolts.

${ }^{a}$ Number of cells in parentheses.

characterize more precisely the nature of synaptic inhibition evoked in XIIts by ipsilateral RAm stimulation, we also bath applied $100 \mu \mathrm{M}$ strychnine in another group of XIIts MNs (Fig. 4). In all 16 cells (nine birds), IPSPs were abolished after strychnine application alone, and EPSPs were again unmasked (Table 2) (mean IPSP amplitude, $-3.5 \pm 0.4 \mathrm{mV}$; mean EPSP amplitude, $\left.+4.8 \pm 0.7 \mathrm{mV} ; t_{(15)}=-8.51 ; p<0.001\right)$. Although strychnine completely blocked RAm-evoked IPSPs in XIIts MNs, it did not alter XIIts MN membrane potential or input resistance (Table 2). These results suggest that synaptic inhibition evoked in XIIts by ipsilateral RAm stimulation is mediated primarily via glycine receptors, although the additional possibility that GABA receptormediated inhibition is present but masked by strong excitation (i.e., in the presence of strychnine) cannot be ruled out at this stage. Finally, we were also interested in knowing whether the onset latencies differed between IPSPs and EPSPs evoked in the same cells, possibly reflecting monosynaptic versus polysynaptic transmission. Similar PSP onset latencies were noted before and after strychnine treatment (IPSP latency, $2.2 \pm 0.2 \mathrm{msec}$; EPSP latency, $1.8 \pm 0.2 \mathrm{msec} ; n=16$ cells; $p=0.13 ; t_{(15)}=1.61$ ), suggesting that both inhibitory and excitatory responses evoked in XIIts by RAm stimulation are mediated by monosynaptic pathways.

Interestingly, in the presence of either GABA and glycine receptor antagonists or glycine receptor antagonists alone, stimulation in RAm could evoke prolonged excitatory responses in XIIts in the form of action potential trains or long-lasting subthreshold depolarizations (Fig. 5). The amplitude and duration of these excitatory responses were highly dependent on the postsynaptic membrane potential, and they diminished in amplitude and became highly phasic when the impaled cell was held in a hyperpolarized state via tonic current injection (Fig. $5 A, B$, Table 2) (control mean EPSP amplitude, $4.4 \pm 0.8 \mathrm{mV}$ at a mean $V_{\mathrm{m}}$ of $-63 \pm 1.8 \mathrm{mV}$; hyperpolarized mean EPSP amplitude, $2.9 \pm$ $0.6 \mathrm{mV}$ at a mean $V_{\mathrm{m}}$ of $-82.9 \pm 3.7 \mathrm{mV} ; t_{(7)}=3.46 ; p=0.01$; mean net negative DC inject $=-0.6 \pm 0.09 \mathrm{nA} ; n=8$ cells from 5 birds).

The voltage dependence of EPSPs evoked in XIIts is suggestive of postsynaptic NMDA receptor activation on the impaled motor neuron, an idea that we confirmed by blocking the slow component of the EPSP with the NMDA receptor blocker D-amino-5phosphopentanoic acid (APV; $100 \mu \mathrm{M}$ ). In the presence of bathapplied strychnine, subsequent puffer pipette application of APV to XIIts significantly reduced the overall amplitude of the evoked EPSP (Figs. 5 B, C, 6, Table 2) (mean EPSP amplitude before APV, $5.2 \pm 0.8 \mathrm{mV}$; mean EPSP amplitude in APV, $3.5 \pm 0.7 \mathrm{mV}$; $t_{(7)}=5.26 ; p=0.0012 ; n=8$ cells from 7 birds). These effects on EPSP amplitude were not accompanied by significant changes in membrane potential (Table 2). Suitably long recordings were obtained to wash out the APV effect in four of these cells (Fig. 5C). The mean EPSP amplitudes before APV $(4.7 \pm 1.1 \mathrm{mV})$ and after drug washout $(5.3 \pm 0.8 \mathrm{mV})$ did not differ significantly from
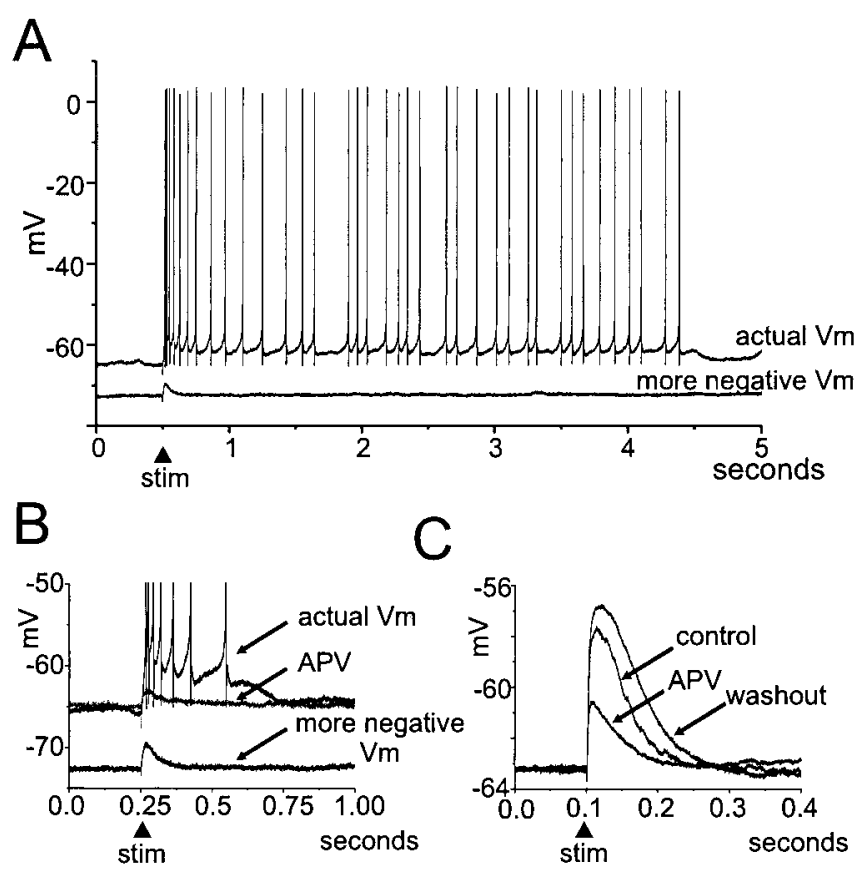

Figure 5. In the presence of glycine receptor blockers, electrical stimulation in ipsilateral RAm evoked EPSPs in XIlts MNs mediated in part by NMDA receptors and which were sufficient to translate single EPSPs into a sustained action potential discharge. $A$, A single EPSP could trigger sustained trains of action potentials at the actual resting potential of the cell (actual Vm) but was rendered highly phasic and entirely subthreshold when the impaled XIIts MN was slightly hyperpolarized via tonic negative current injection through the recording electrode (more negative $\mathrm{Vm}$ ). $B$, The slow portion of the EPSP evoked by RAm stimulation in brain slices bathed in strychnine had the voltage-dependence characteristic of NMDA receptor-mediated transmission and was strongly attenuated by hyperpolarization of the postsynaptic XIIts membrane. The EPSP evoked by RAm stimulation is displayed at the actual resting potential of the cell (topmost trace; actual Vm) or at a slightly more negative membrane potential (bottom trace; more negative $\mathrm{Vm}$ ) achieved by passing tonic current through the recording electrode. The EPSP triggered a train of action potentials at the more positive membrane potential. Subsequent treatment with the NMDA receptor blocker D-APV reduced the EPSP in a manner resembling postsynaptic hyperpolarization, localizing the NMDA receptors to the impaled cell. C, The slow portion of the EPSP was blocked reversibly by applying NMDA receptor antagonists to XIlts. EPSPs that were collected before and during application of the NMDA receptor antagonist D-APV to XIIts are shown superimposed (see Materials and Methods). These results suggest that NMDA receptors on the XIlts MN are activated by lateral medullary inputs. Raw traces are shown in $A$ and $B$, and averages of three to six traces are shown in C. Stim, Stimulation time.

one another $\left(t_{(3)}=-0.68 ; p=0.55\right)$, whereas both were significantly larger than the mean EPSP amplitude measured in the presence of APV $\left(2.7 \pm 0.7 \mathrm{mV} ; t_{(3)}=3.78, p=0.03\right.$ and $t_{(3)}=$ $-4.99, p=0.01$, respectively). Ultimately, the similar effects of postsynaptic hyperpolarization and APV treatment (Figs. 5B, 6) on the slow component of the EPSP suggest that axons arising from or traveling through the lateral medulla activate NMDA receptors on XIIts MNs. Presumably, the synaptic response evoked in the presence of D-APV was mediated by non-NMDA glutamate receptor types. 


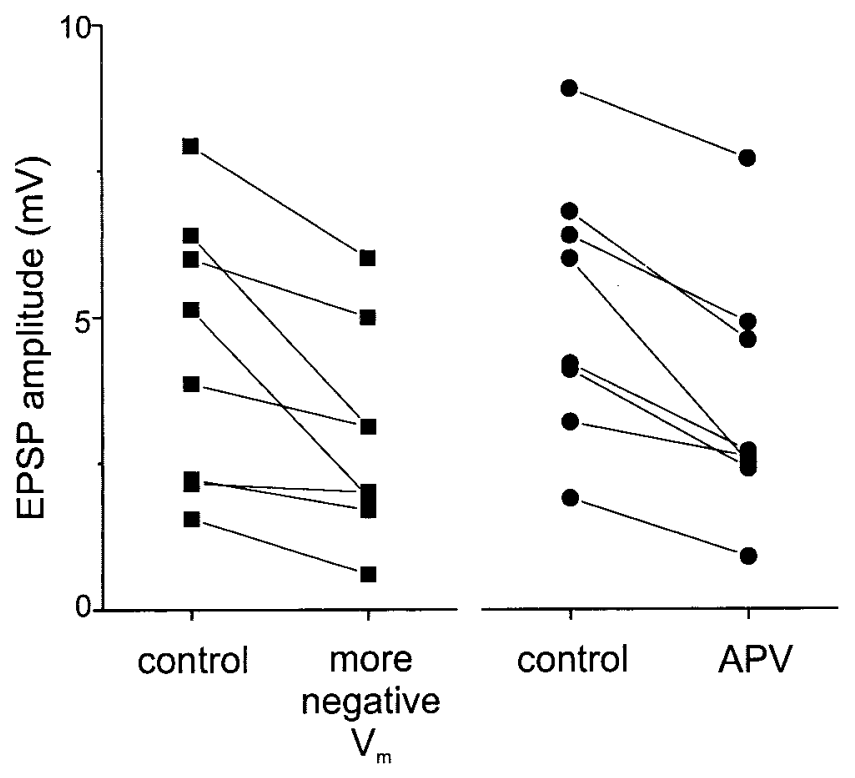

Figure 6. Scatterplots of EPSP amplitude before (control) and with the impaled cell held in a hyperpolarized state (left; more negative $V_{m}$ ) or treated with D-APV (right; APV). With either treatment, EPSPs were attenuated to a similar degree (Table 2).

To determine whether PSPs evoked in XIIts by electrical stimulation of the lateral medulla were caused by synaptic inputs derived from cell bodies in RAm or instead from fibers of passage arising from more rostral sources of input to XIIts (Wild, 1994, 1997), we focally applied glutamate (10 mM dissolved in ACSF) at the electrical stimulation site in ipsilateral RAm. As with the electrical stimulation, synaptic responses from XIIts MNs were evoked by glutamate application from hot spots typically directly under the simulating electrode. In 12 cells from six birds, glutamate application elicited membrane potential changes in the impaled XIIts $\mathrm{MN}$, and the initial component of the chemically evoked response was always of the same sign as that of the synaptic response evoked by electrical stimulation at the same site (Fig. 7) (hyperpolarizing responses were seen in 10 cells, and depolarizing responses were seen in 2 others). Furthermore, as with electrical stimulation, we found in four other cells that focal glutamate application that initially evoked hyperpolarizing responses from XIIts MNs in normal ACSF subsequently evoked depolarizing, excitatory responses after bath application of strychnine (Fig. 7) $(n=4$ cells). Taken together, these results suggest that different populations of neurons located within the lateral medulla supply XIIts MNs with inhibitory and excitatory synaptic input.

\section{In vivo properties of XIIts MNs}

The brain slice recordings that we made suggest that a dominant synaptic input to XIIts from respiratory areas in the lateral medulla activates glycine receptor-mediated inhibition but that this inhibition often masks an underlying excitatory component. Because previous extracellular recordings in XIIts and from the tracheosyringeal nerve made in vivo detected expiratory linked firing and left the synaptic basis of such activity unresolved, we were interested in relating in vitro to in vivo synaptic function in XIIts. Therefore, we made additional intracellular recordings from XIIts MNs in vivo, assessing their intrinsic properties, the synaptic events underlying their respiratory activity, and their modulation by synaptic inputs from the telencephalon.

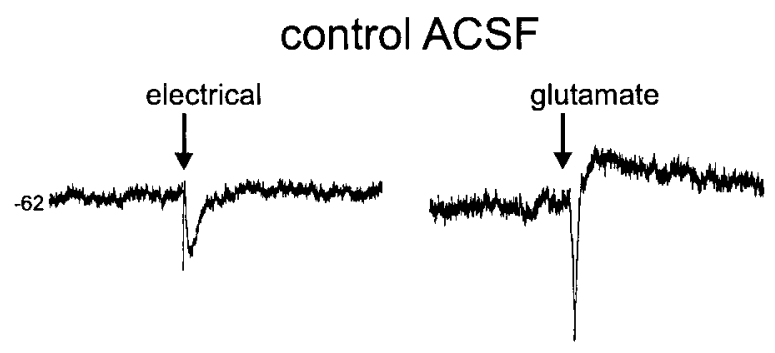

strychnine

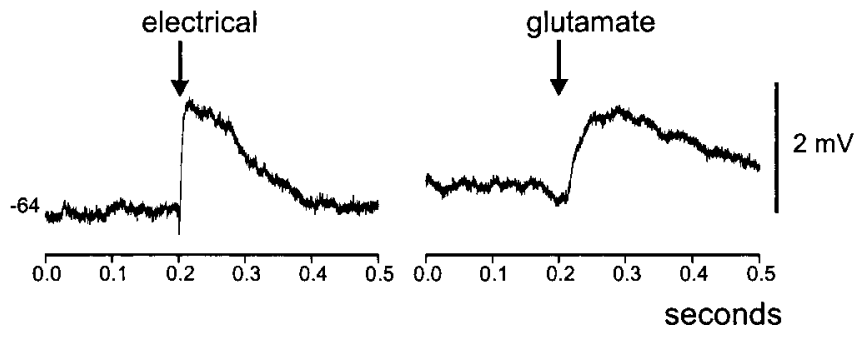

Figure 7. EPSPs and IPSPs evoked in XIIts MNs from lateral medullary stimulation are attributable to cell bodies local to RAm. In control ACSF (top), electrical stimulation and glutamate application in the same spot in the lateral medulla evoked similar inhibitory responses in this XIlts MN. After strychnine application (bottom), which abolished the inhibitory responses, the same stimulation protocol evoked depolarizing, excitatory responses. An arrow marks the time of electrical (left) or chemical ( glutamate; right) stimulation in each case. Traces shown are averages of three to six individual records.

\section{In vivo XIIts $\mathrm{MN}$ intrinsic properties}

The intrinsic properties of XIIts MNs were remarkably similar to those that we measured in brain slices. In vivo, XIIts MNs had average resting potentials of $-60 \pm 4 \mathrm{mV}$ (mean $\pm \mathrm{SEM} ; n=6$ cells from 5 birds) and average input resistances of $49 \pm 11 \mathrm{M} \Omega$ in vivo (Table 1$)$ ( $n=5$ cells from 5 birds) and produced spontaneous, rhythmic action potential trains entrained to the expiratory phase of respiration (see below). Positive current pulses injected through the recording electrode also evoked action potential trains with mean and instantaneous firing frequencies resembling those seen in XIIts MNs recorded in brain slices (Fig. 2D,E). Unlike XIIts MNs recorded in vitro, brief interruptions in action potential discharge were occasionally observed in vivo during the injection of small positive currents (Fig. 2C, arrow), possibly reflecting inspiratory-linked suppression of XIIts MN activity. AHPs and ADPs were observed in all six cells tested with positive currents (six of six cells from five birds), and anomalous rectification was seen in a subset of cells that were examined (three of six cells in vivo) (Table 1).

\section{In vivo characterization of XIIts $\mathrm{MN}$ respiratory activity}

To examine the synaptic events underlying respiratory activity in XIIts MNs, we made intracellular recordings from them while simultaneously monitoring respiration using abdominal EMGs. A comparison of intracellular and EMG records revealed a highly regular pattern of action potential discharge in XIIts MNs that was predominantly in phase with the activity in abdominal expiratory muscles (Fig. 8) (12 cells from two birds). Firing persisted throughout expiration and ceased at the offset of the expiratory EMG (i.e., during inspiration).

Although the initial inspection of intracellular traces showed that XIIts MNs were active during expiration and quiescent during inspiration, the synaptic events underlying the respiratory rhythm were not readily apparent because of sustained action potential discharge during expiration. To detect underlying syn- 


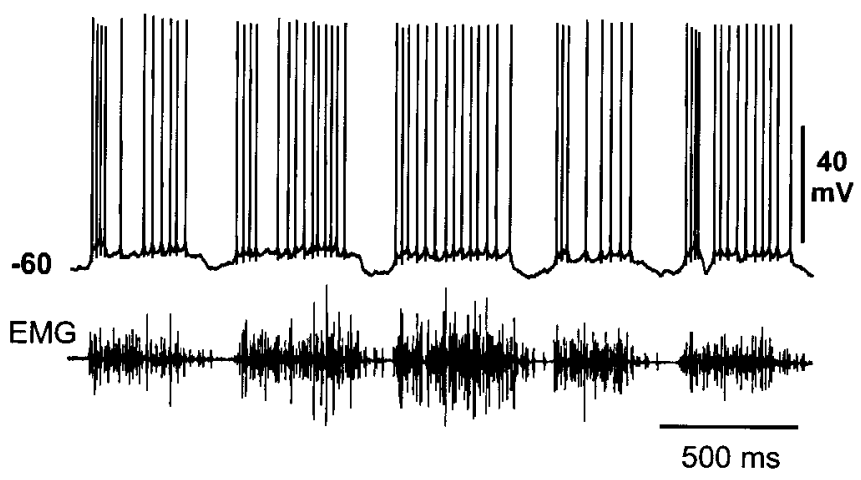

Figure 8. Xllts motor neurons display a pronounced respiratory rhythm. An in vivo intracelIular recording from an XIIts MN was used to monitor its membrane potential, whereas a simultaneous EMG was obtained from abdominal expiratory muscles to measure respiratory activity. Xllts MN firing was always in phase with the expiratory EMG.

aptic events, we varied the membrane potential of XIIts MNs by injecting depolarizing or hyperpolarizing DC current through the recording electrode (approximately $\pm 1 \mathrm{nA}$ ). When XIIts MNs were hyperpolarized below their normal resting potential, their pattern of activity changed markedly; in this more negative state, XIIts MNs produced very phasic bursts of only a few action potentials at the onset of expiration (Fig. 9). This pattern of activity in a hyperpolarized state was suggestive of a strong excitatory input onto XIIts MNs at expiration onset. When XIIts MNs were held at or slightly above their actual resting potential, they produced trains of action potentials that began in phase with the onset of expiration and terminated with expiratory EMG offset. However, in neither hyperpolarized nor depolarized states were we able to detect inhibitory synaptic events underlying expiration offset.

\section{In vivo responses of XIIts MNs to song playback}

In addition to receiving respiratory-related inputs from the lateral medulla, XIIts MNs are the target of direct synaptic input from the telencephalic song nucleus RA. In urethaneanesthetized zebra finches, auditory presentation of the BOS potently excites telencephalic song nuclei, including RA (Margoliash and Konishi, 1985; Doupe and Konishi, 1991; Vicario and Yohay, 1993). Such playback also activates XIIts neurons (Williams and Nottebohm, 1985), likely via the projections that they receive from RA. Furthermore, a recent study showed the BOSevoked activity of RA neurons resembles their activity during singing (Dave and Margoliash, 2000), suggesting that playback also triggers behaviorally relevant activity patterns in XIIts.

To confirm that the song-evoked responses observed in XIIts were derived from the telencephalic vocal control system, we made multiunit extracellular recordings of XIIts activity in response to BOS playback and then reversibly inactivated $\mathrm{HV}_{\mathrm{c}}$, the auditory afferent of RA (Doupe and Konishi, 1991; Mooney, 1992; Vicario and Yohay, 1993). Before GABA application (0.25 $\mathrm{M}$ in ACSF) to $\mathrm{HVc}$, forward BOS presentation evoked action potential discharge in XIIts MNs; GABA application to HVc reversibly abolished these responses (Fig. 10) (three sites in two birds).

Although the inactivation experiments indicate that inputs from the telencephalon are necessary to drive song-evoked firing in XIIts, they do not rule out the existence of other auditory inputs that are insufficient on their own to drive XIIts MNs to spike threshold. Temporal sensitivity to the BOS, as manifested as a strong bias in response strength to forward over reverse BOS slight tonic depolarization

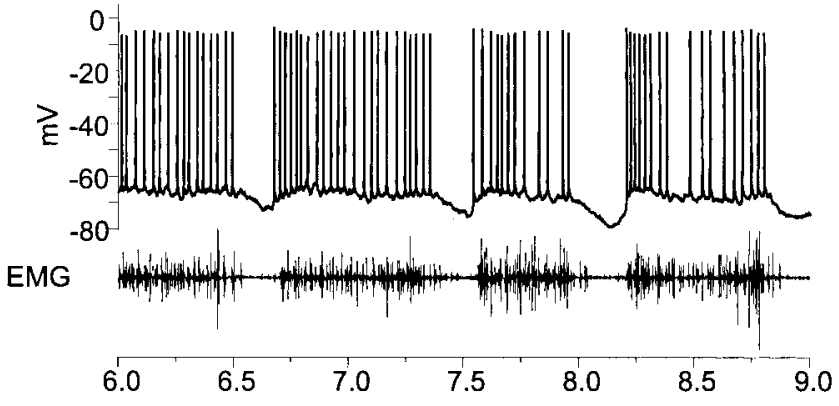

slight tonic hyperpolarization

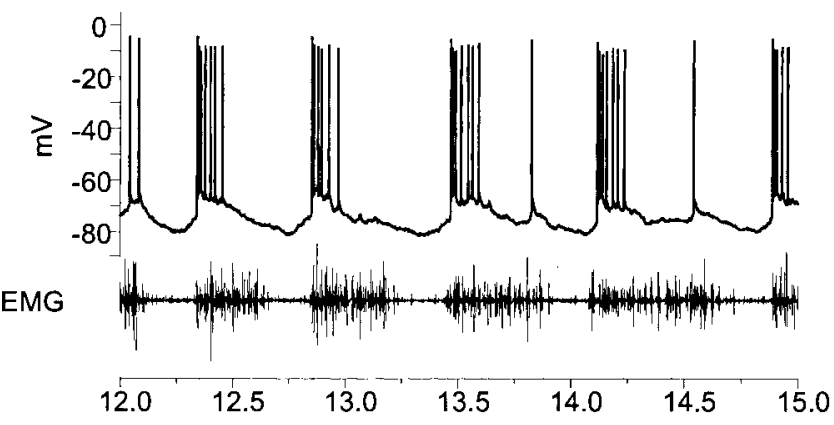

greater tonic hyperpolarization

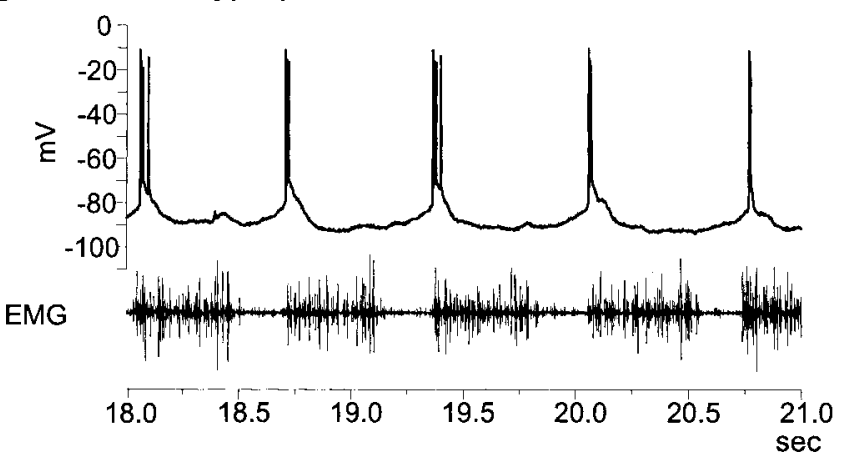

Figure 9. Membrane potential manipulations reveal phasic excitation at expiratory EMG onset. An in vivo intracellular recording $(\mathrm{mV})$ from an XIIts MN and a simultaneous abdominal muscle EMG (EMG) were used to measure the nature of respiratory activity in XIlts. During these recordings, the XIIts MN membrane potential was either slightly depolarized (top) or moderately or more strongly hyperpolarized (middle and bottom) by injecting positive or negative currents through the recording electrode. When slightly depolarized from its resting membrane potential value with positive current $(+0.5 \mathrm{nA}$, top), the neuron showed only gradual membrane hyperpolarization (but abrupt firing rate suppression) during the inspiratory phase. When moderately or more substantially hyperpolarized from its resting membrane potential with tonic negative current passed through the recording electrode $(-1.25 \mathrm{nA}$, middle; -1.5 $\mathrm{nA}$, bottom), the neuron displayed subthreshold excitation occurring abruptly at the onset of expiration. No membrane potential movements were noted at the offset of expiratory EMG activity.

playback, is one feature that distinguishes auditory responses in RA from many other auditory areas. When presented with these two stimuli, all XIIts MNs were excited significantly by forward but not reverse BOS playback (Figs. 11, 12) (8 intracellular recordings; 10 extracellular multiunit recordings; mean response strength to forward BOS: intracellular = 29.45; extracellular = 5.56) suprathreshold responses; no significant excitatory responses were evoked by reverse BOS). Firing rate responses to the forward and reverse BOS recorded either intracellularly or extracellularly showed an overwhelming bias toward the forward stimulus (Fig. $11 A$ ) [mean BOS $Z$-score intra $=4.0 \pm 0.7$; mean reverse 


\section{Pre-inactivation}
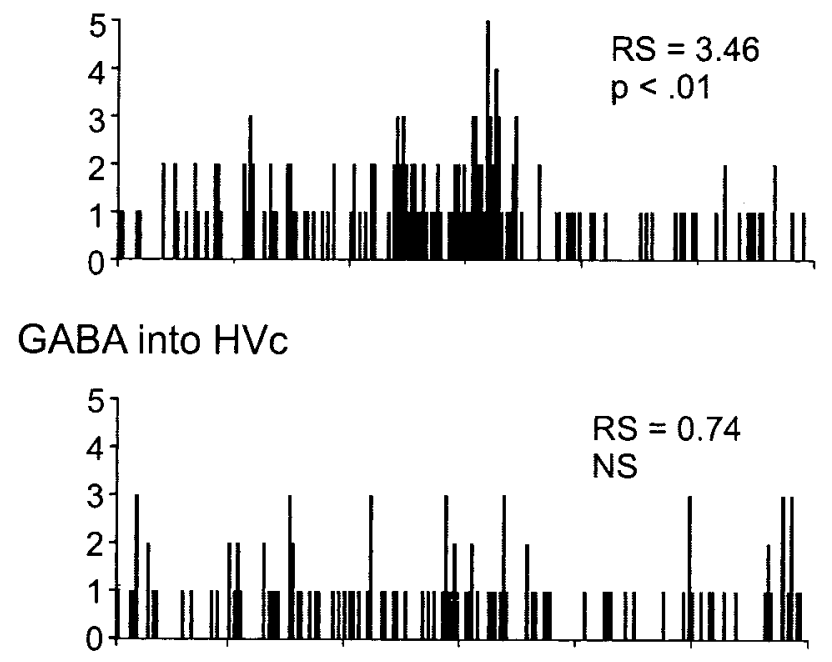

\section{Post-inactivation}

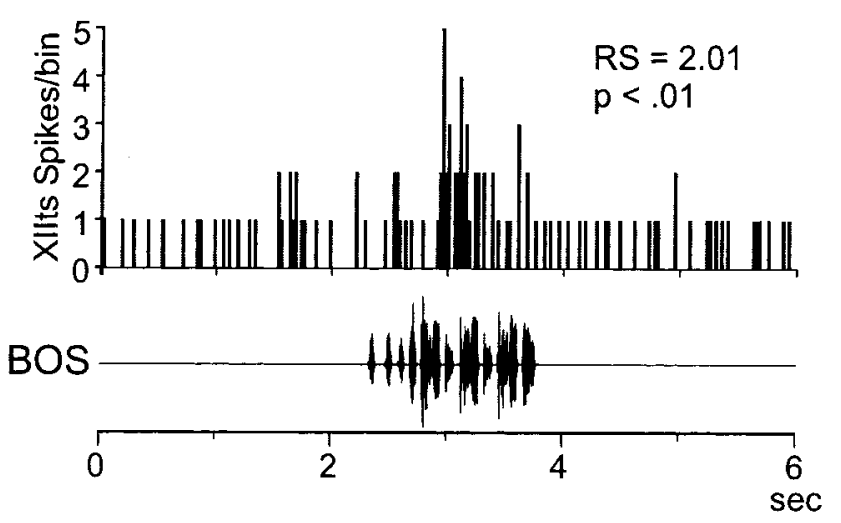

Figure 10. The BOS playback-evoked responses in XIIts depend on activity in the telencephalic song nucleus HVc. A multiunit extracellular recording was made in XIIts, and baseline responses to BOS playback (shown as an oscillogram at the bottom) were collected [top, Preinactivation; 10 stimulus iterations; responses are shown as action potential PSTHs (XIIts spikes) bin)]. The telencephalic song nucleus HVc was then inactivated by injecting it with concentrated GABA ( $0.25 \mathrm{~m}$ in $0.9 \%$ saline) ejected through a puffer pipette placed stereotactically in the nucleus. BOS playback failed to excite XIlts MNs when HVc was inactivated with GABA (middle, $G A B A$ into HVC). Reponses to BOS playback could again be detected within several minutes after GABA application to HVc (bottom, Post-inactivation). Response strengths ( $R S$ ) and significance levels are given for each condition.

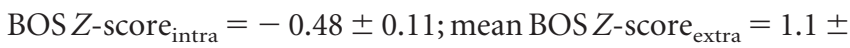
0.2 ; mean reverse $\mathrm{BOS} Z$-score extra $=-0.04 \pm 0.1$; paired $t$ tests; BOS versus reverse (REV) BOS, for intracellular $\left(t_{(7)}=5.87, p<\right.$ $0.001)$ and extracellular $\left(t_{(9)}=5.55, p<0.001\right)$ suprathreshold responses]. To inspect underlying synaptic events, we tonically hyperpolarized impaled XIIts MNs, accentuating subthreshold depolarizations evoked by forward BOS playback (Figs. 12, 13) [mean BOS $Z$-score $_{\text {intra }}=2.21 \pm 0.67$; mean reverse BOS $Z$-score intra $_{\text {in }}=-0.06 \pm 0.20$; paired $t$ test; BOS versus REV BOS, for intracellular subthreshold responses $\left(t_{(6)}=4.154, p=\right.$ 0.006)]. Although reverse BOS playback elicited neither subthreshold nor suprathreshold excitatory responses (Figs. 11, 12, $13 ; n=5$ cells), it did suppress firing in one cell. A direct comparison of selectivity for the forward over reverse BOS stimulus, quantified as a $d^{\prime}$ value, indicated that, as a population, XIIts MNs display both action potential and subthreshold responses highly selective for the forward song (Fig. 11B) (suprathreshold:

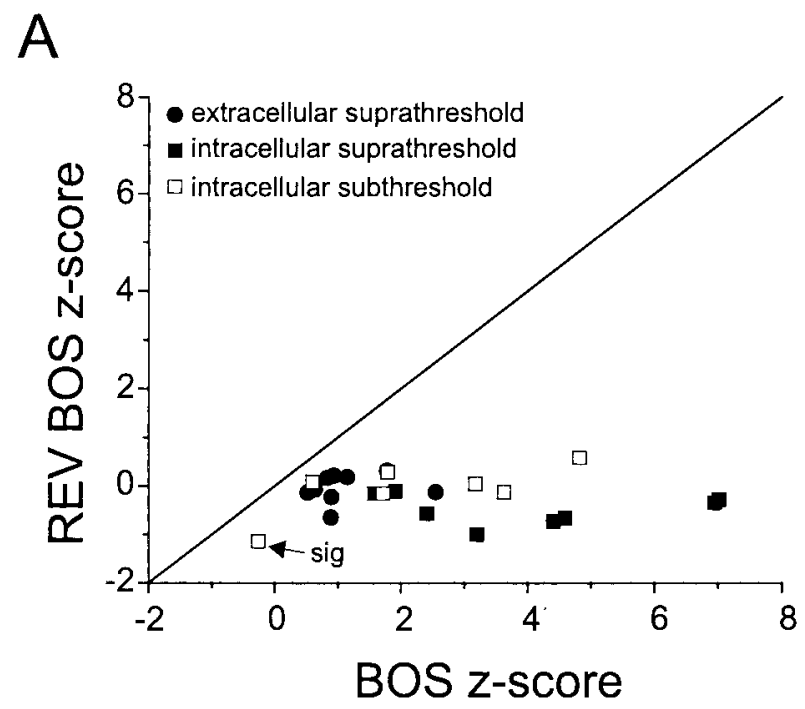

B

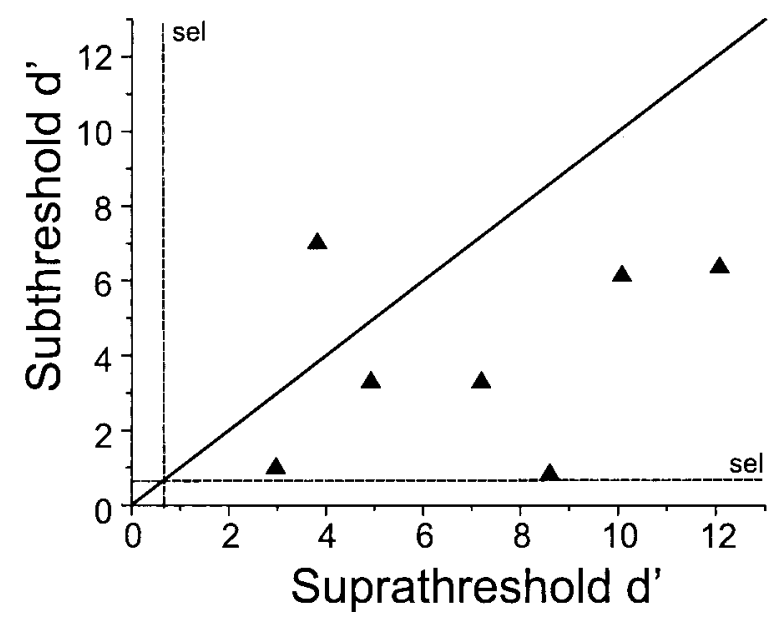

Figure 11. XIlts MNs show subthreshold and suprathreshold responses that are highly selective for forward over reverse BOS playback. A, Z-scores of forward ( $X$-axis) and reverse BOS (REV BOS) ( $y$-axis) evoked suprathreshold responses (from extracellular and intracellular recordings; filled circles and filled squares, respectively) and subthreshold responses (from intracellular recordings; open squares) show an overwhelming bias to the forward stimulus, indicative of strong temporal sensitivity to the BOS. All forward BOS responses were highly significant; an arrow notes the only significant reverse BOS response, which involved firing rate suppression. $B$, Within-cell comparisons of subthreshold versus suprathreshold $d^{\prime}$ values obtained from intracellular records indicate that XIIts MNs are more selective for forward over reverse BOS at a suprathreshold than at the subthreshold level (dashed lines indicate the lower bounds for selective BOS responses).

mean BOS $d^{\prime}{ }_{\text {intra }}=7.05 \pm 1.10$, mean BOS $d^{\prime}{ }_{\text {extra }}=1.79 \pm 0.30$; subthreshold: mean $\left.d^{\prime}{ }_{\text {intra }}=3.97 \pm 0.97\right)$. Along with the effects of HVc inactivation, the highly selective responses seen in XIIts suggest that RA is likely to be its sole source of auditory input.

To characterize further the nature of telencephalic inputs to XIIts, including their possible interaction with respiratory inputs, we recorded BOS-evoked activity either extracellularly or intracellularly from XIIts MNs while monitoring the respiratory cycle via abdominal expiratory EMGs (Fig. 12). Although the net effect of BOS-evoked telencephalic drive to XIIts is clearly excitatory, as evinced in both extracellular and intracellular records, intracellular records also revealed that BOS-evoked excitation could be preceded by a brief $(\sim 50 \mathrm{msec})$ hyperpolarizing movement of the 

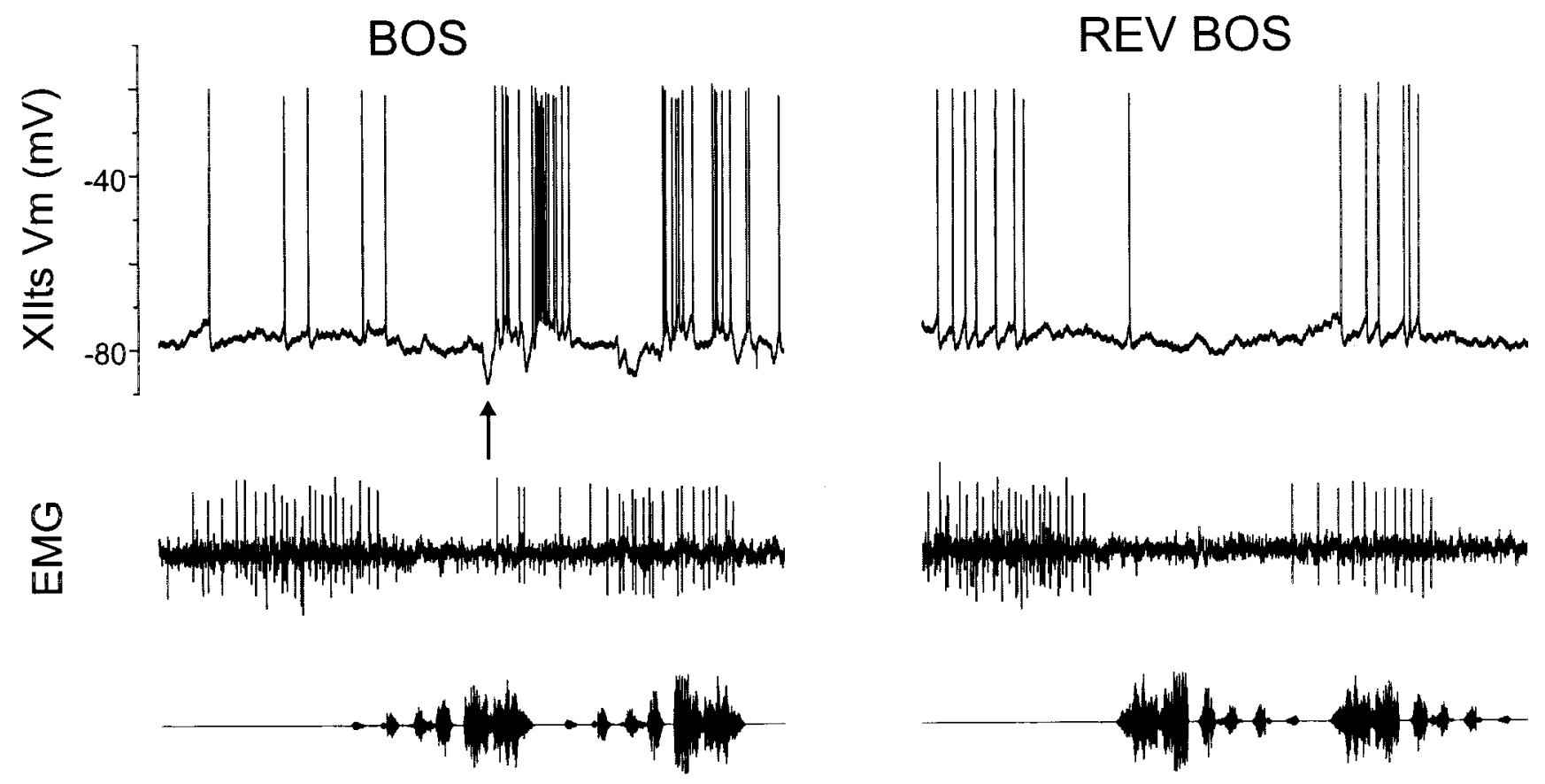

$0.5 \mathrm{sec}$

Figure 12. Forward and not reverse playback of the bird's own song (BOS) strongly excites Xllts MNs. An in vivo intracellular recording from an XIlts MN reveals its membrane potential responses [XIIts Vm (mV)] to playback of the BOS and reverse BOS (REV BOS); a corresponding expiratory EMG is shown below each intracellular trace. Strong excitatory suprathreshold responses accompanied by subthreshold depolarizations were detected in response to forward, but not reverse, BOS playback. Unlike Xllts activity in the absence of song playback, hyperpolarizing events can be seen (top trace, marked by an arrow at the onset of the BOS-evoked response), and strong excitation could occur out of phase with the expiratory EMG. The absence of subthreshold responses to reverse BOS (Figs. 11, 14) in XIlts indicates that its auditory afferent does not generate action potentials to this stimulus. Such behavior is characteristic of neurons in RA, the telencephalic afferent of Xllts, consistent with the idea that RA is the source of Xllts auditory responses.

membrane potential (five cells from one bird). Such hyperpolarizing events were observed both in individual raw membrane potential records and in three of the five cells in the averaged median filtered membrane potential traces (Figs. 12, 13) and were characterized by sharp onsets and negativities below membrane potential values before the stimulus (Fig. 13B).

The hyperpolarizing events that we observed are consistent with the idea that, in addition to directly exciting XIIts, RA also excites inhibitory cells in RAm that innervate XIIts. More direct evidence of the influence of RA on RAm was noted by monitoring abdominal muscle EMGs during song playback: in two birds, forward but not reverse BOS playback augmented the expiratory EMG (Fig. 14). Another apparent and possibly related effect of forward and reverse BOS playback on RAm was to entrain expiratory activity: PSTHs of abdominal expiratory EMG activity constructed from multiple stimulus iterations revealed pronounced troughs and peaks deviating from the prestimulus baseline (Fig. 14) ( $n=2$ birds).

We also assessed whether the response of a cell to BOS differed if the playback immediately followed an expiratory event, as measured by heightened EMG activity, or instead by a period of muscular inactivity, presumed to reflect inspiration. In four of five cells, response strength was higher when expiration immediately preceded playback, and the mean response strength was slightly higher in the "expiratory" versus "inspiratory" group, although this effect did not achieve significance $\left(R S_{\text {BOSexp }}=29.6 \pm 7.0 \mathrm{vs}\right.$ $\left.R S_{\text {BOSinsp }}=23.9 \pm 9.3 ; t=-1.9 ; \mathrm{df}=4 ; p=0.13\right)$.

To assess relative levels of synaptic activity in XIIts during BOS-evoked excitation and subsequent respiratory entrainment, we applied brief negative current pulses $(-0.5 \mathrm{nA})$ through the recording electrode before, during, and after playback. The resultant voltage deflections diminished transiently near the peak of the forward BOS-evoked depolarizations, consistent with the idea that forward BOS playback augments excitatory synaptic drive to XIIts MNs (Fig. 14) (relative impedance change $-8.1 \pm$ $1.9 \mathrm{M} \Omega$, a $31 \%$ decrease; $n=3$ cells). Furthermore, in almost all (four of five) of the cells that we tested, decreases in postsynaptic impedance were detected at the onset of the entrained expiratory onset but not during inspiration (relative impedance change $-2.4 \pm 0.3 \mathrm{M} \Omega$, a $10 \%$ decrease; $n=4$ cells). The impedance of one cell decreased at the trough of the entrained expiratory EMG (i.e., at inspiration). These impedance changes suggest that synaptic activity in XIIts increases prominently during BOS playback and expiration but not during inspiration.

\section{Discussion}

In both anesthetized and awake nonsinging zebra finches, XIIts MNs fire action potentials predominantly in phase with expiration, as measured extracellularly in the nucleus or nerve or by syringeal EMGs (Manogue and Paton, 1982; Williams and Nottebohm, 1985; Vicario, 1991a) and confirmed here intracellularly. Although previous studies left open whether this rhythm results from expiratory-linked excitation or inspiratory-linked inhibition, or both, we observed that the major component of this rhythm involves expiratory-linked excitation. Hyperpolarization of the XIIts MN revealed phasic subthreshold depolarizations locked to expiratory onset and an absence of membrane potential movement at expiratory offset. Furthermore, in cases showing expiratory EMG entrainment to song playback, postsynaptic impedance decreases (likely reflecting enhanced synaptic 


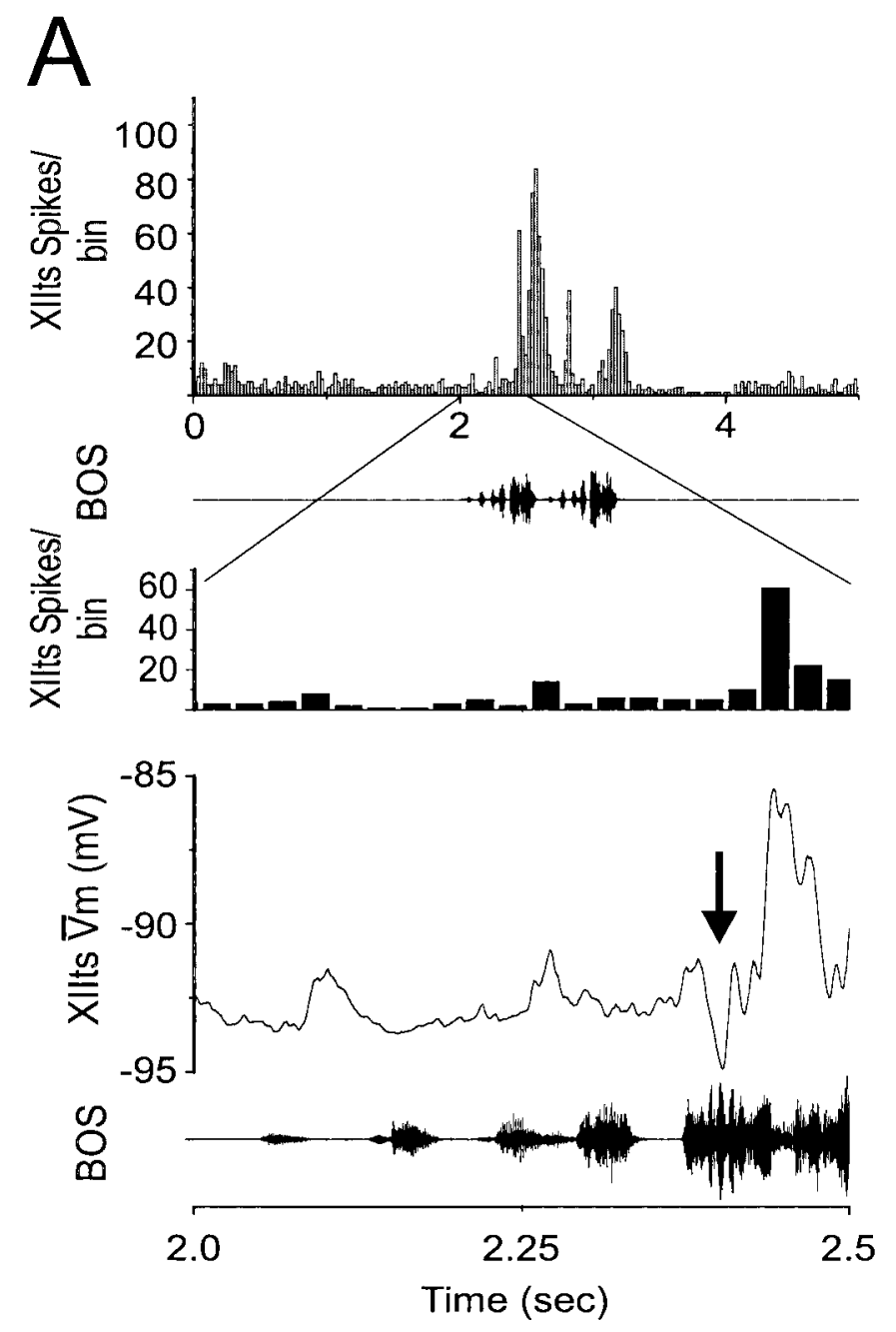

B
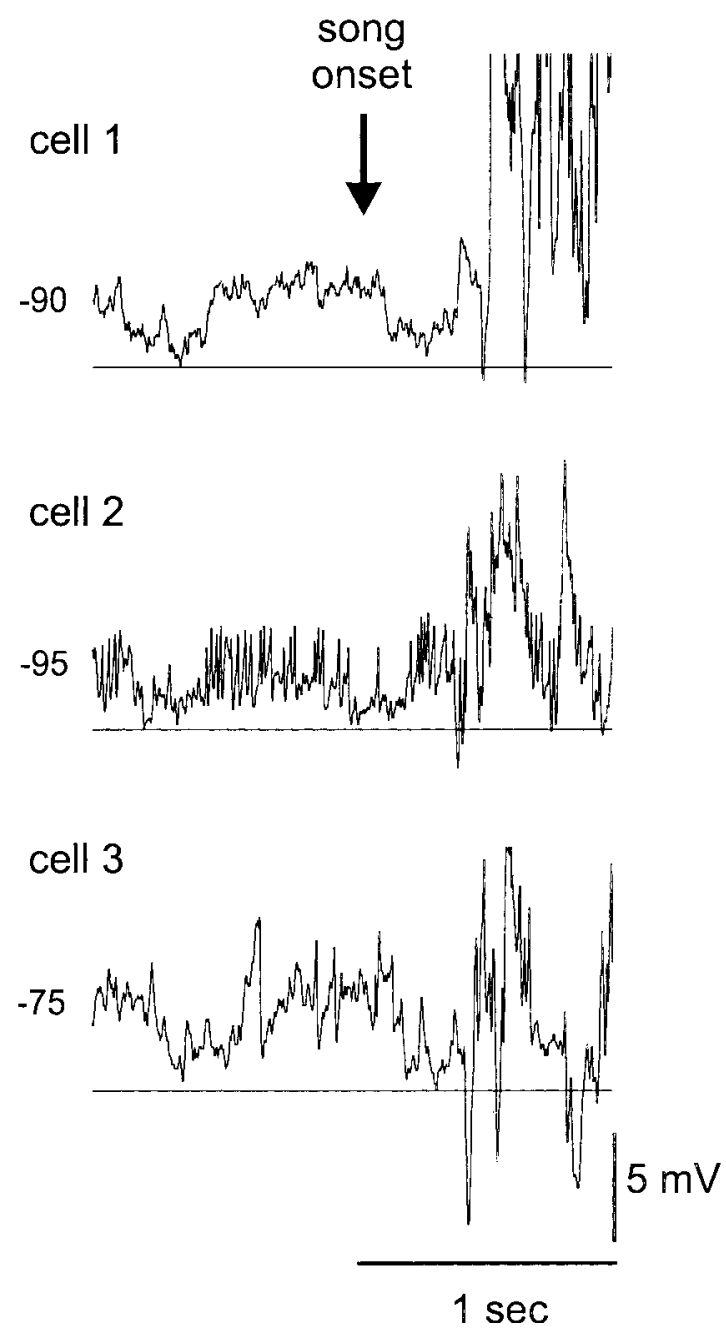

Figure 13. Excitatory BOS-evoked responses in XIlts MNs could be preceded by subthreshold hyperpolarizations, suggestive of auditory-evoked inhibition. $A$, An in vivo intracellular recording from an XIIts MN revealed that strong excitatory responses were evoked by BOS playback (action potential responses to the entire BOS playback are shown as a PSTH at the top). An expanded region near BOS onset (shown as an oscillogram at the bottom) shows the PSTH and the averaged median-filtered membrane potential record aligned to the stimulus. Note that a sharp hyperpolarization precedes the initial volley of action potentials. This feature could be mediated by inhibition onto the XIlts MN driven by RA axons that innervate lateral medullary areas including RAm and PAm (Fig. 1). $B$, Individual, median-filtered membrane potential traces from three cells [including the cell shown in $A$ (cell 1 )] show that BOS-evoked hyperpolarizations exceeded the negativities associated with the resting respiratory rhythm (marked by a horizontal line), even when the cell was held at relatively hyperpolarized potentials with tonic negative current (cell 1 and cell 2; "resting" membrane potential in millivolts is indicated to the left of each trace). Song onset marks the beginning of the BOS playback.

activity) commonly were detected in XIIts MNs during the subsequent expiratory phase but not during inspiration. Although we cannot exclude more cryptic forms of inspiratory-linked inhibition in XIIts, these results emphasize that expiratory-linked excitation dominates the XIIts respiratory rhythm. During singing, expiratory excitation of XIIts could serve as an "and" gate (Manogue and Paton, 1982), at least for MNs involved with syringeal adduction (Goller and Suthers, 1996a), thereby precisely coordinating expiration and vocal output (Hartley and Suthers, 1990).

In direct contrast to a model of purely expiratory-driven XIIts excitation, however, we found in vitro that electrical stimulation in a lateral medullary area corresponding to RAm evoked IPSPs from the majority of XIIts MNs. These IPSPs were mediated primarily by glycine receptors and reversed at relatively negative potentials, consistent with a potassium current. Moreover, glutamate application to RAm also evoked IPSPs, suggesting that they were mediated by RAm neurons and not by fibers passing through RAm to XIIts. Additional respiratory-related inputs to XIIts not characterized here might arise from more rostral brainstem sources, including the inspiratory-related PAm (Reinke and Wild, 1998) and the ventrolateral parabrachial pontine nucleus (Wild et al., 1990).

Despite the strong inhibitory influence of RAm on XIIts observed in the slice, in vitro recordings also point to RAm as the likely source of expiratory-linked excitation to XIIts. When inhibition to XIIts was blocked, electrical and chemical stimulation of RAm routinely evoked EPSPs in XIIts MNs. These results suggest that two functionally distinct RAm neuron types innervate XIIts, an excitatory one driving expiratory-linked XIIts firing and an inhibitory one not strongly recruited during normal respiration (Fig. 15). Another idea is that RAm neurons are exclusively excitatory and synapse on both XIIts MNs and inhibitory neurons in or near XIIts, although two observations make this less likely. 
First, EPSPs and IPSPs evoked in XIIts by electrically stimulating RAm had similarly short latencies, inconsistent with an interposed inhibitory neuron; second, we detected only one XIIts cell type (i.e., MNs), suggesting that RAm axons in XIIts terminate on MNs and not on interneurons.

Regardless, the activation of NMDA receptors on XIIts MNs by the excitatory synaptic input, coupled with the intrinsic properties of these cells, may enable XIIts MNs to translate more phasically active inputs, such as those detected at expiratory onset or that may arise from RA during singing ( $\mathrm{Yu}$ and Margoliash, 1996), into prolonged action potential trains. The intrinsic properties of XIIts MNs seem well suited for both breathing and singing. Both in vitro and in vivo, XIIts MNs maintain action potential discharge rates of $\sim 20 \mathrm{~Hz}$. During breathing, this may allow them to brake the rate of airflow during normally active expiration or otherwise to maintain bronchial patency, specifically via their innervation of syringeal muscles controlling the syringeal labia (Goller and Suthers, 1996b). Indeed, songbirds with bilaterally sectioned syringeal nerves audibly wheeze during expiration (Nottebohm, 1968; Vicario, 1991a), likely because of bronchial occlusion by flaccid syringeal membranes. In contrast to resting discharge rates, XIIts MN firing rates increase linearly and steeply with injected current, properties displayed extensively during singing, when XIIts activity rises high above resting levels.

In vivo intracellular recordings from XIIts MNs in the anesthetized bird, coupled with song playback to selectively activate telencephalic song nuclei, provide a useful intermediary between reduced slice preparations and chronic recordings in singing birds. Here we extend an earlier extracellular study (Williams and Nottebohm, 1985) by showing that BOS play-

back strongly depolarizes XIIts MNs and sharply decreases postsynaptic impedance, indicative of excitatory synaptic drive. This excitation is likely driven by RA synapses on XIIts MNs, because inactivation of $\mathrm{HVc}$ (the auditory afferent of RA) (Doupe and Konishi, 1991; Vicario and Yohay, 1993; Mooney, 2000) abolished XIIts responses (Williams and Nottebohm, 1985) and because reverse BOS, which does not excite RA neurons (Yu and Margoliash, 1996; Doupe and Konishi, 1991), failed to evoke subthreshold responses in XIIts MNs. The lack of responses to reverse BOS, a stimulus that can drive subthreshold responses in some song nuclei (namely HVc) (Mooney, 2000), indicates that auditory inputs are highly filtered before reaching XIIts and thus do not reflect connections between auditory brainstem structures and XIIts. However, because RA also contacts the dorsomedial nucleus of the intercollicularis, which in turn innervates XIIts, at least two sources may drive BOS-evoked XIIts activity.
BOS

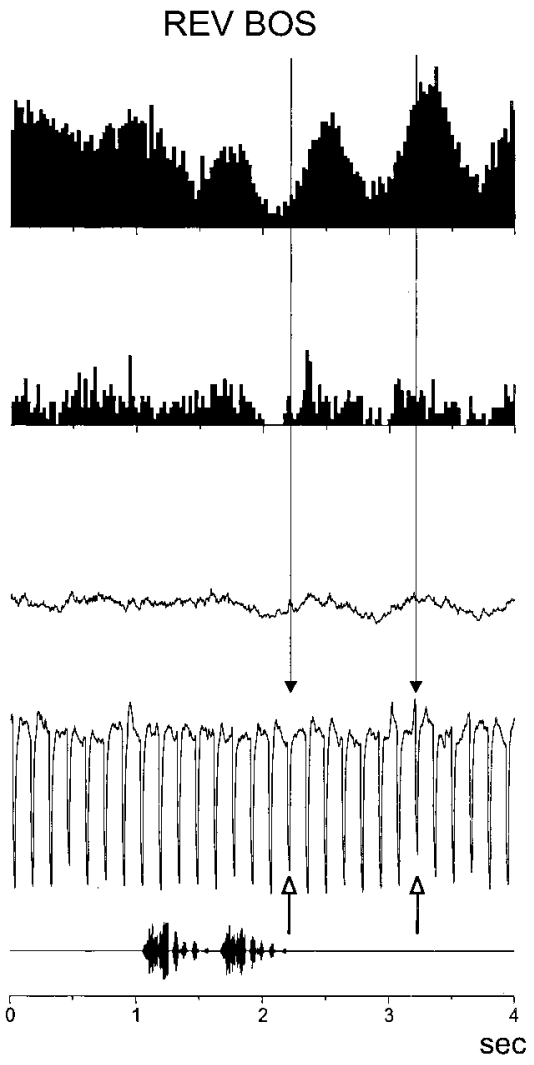

sec

Figure 14. Changes in XIIts MN input impedance during BOS playback and during subsequent respiratory entrainment. Forward (bottom left) and reverse BOS (REV BOS) (bottom right) playback-evoked vocal and respiratory activity, as revealed by simultaneous (he augmentation in XIIts MN (enhanced EMG activity is marked by a solid arrow). Inspiration, reflected as troughs in the EMG, consistently followed forward BOS playback in this case. Such respiratory entrainment also occurred in ponse to reverse BOS playback, although this stimulus did not excite the XIIts neuron (note different $y$-axis scales for the forward BOS-evoked XIlts PSTHs). Postsynaptic impedance decreases, measured as decrements in the amplitude of the DCduring subsequent expiratory phases (marked by open arrows, aligned to XIlts spiking and EMGs with closed arrows). These results iterations each of forward and reverse BOS playback were used to construct the PSTHs of XIlts MN and expiratory EMG activity, whereas 20 iterations were used to generate each of the two median filtered $V_{m}$ values (i.e., current pulses were not injected through the recording electrode on every trial).

Recently, chronic recordings in zebra finches revealed that BOS playback evokes RA activity patterns similar to those produced during singing (Dave and Margoliash, 2000). An extension of this view is that BOS playback can evoke respiratory and vocal activity reminiscent of that in the singing bird. We observed (in two birds) that BOS playback not only excited XIIts but also affected patterning of the expiratory muscle EMG, including distinct peaks of EMG activity preceding the XIIts MN firing. These observations suggest that BOS playback activates both dorsal and ventral regions of RA, ultimately exciting XIIts MNs and RAm bulbospinal expiratory-related premotor neurons (Wild, 1993a,b). How reverse BOS affected expiratory muscle activity is less clear, but it may point to a general arousal effect or other auditory inputs to RAm expiratory premotor neurons in addition to those arising from RA.

Further indications of the influence of RA on RAm was the unexpected finding that XIIts MN excitation could be preceded 


\section{Normal Respiration}

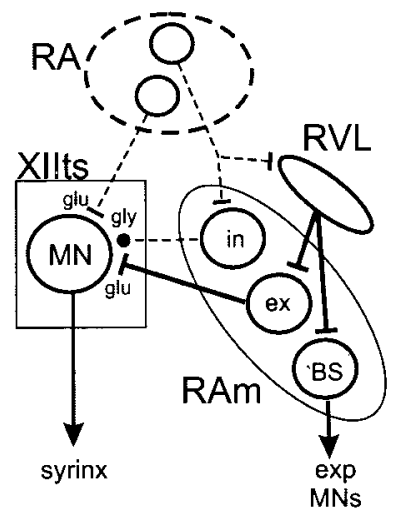

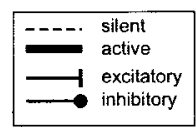

Figure 15. Partial schematic of ipsilateral pathways involved during normal (quiet) respiration (left) and song playback/singing (right). During normal respiration, Xllts activity is controlled primarily by expiratory-linked RAm neurons, whereas telencephalic influence from RA is negligible (dashed lines). The respiratory rhythm, generated in or channeled through the rostral nucleus of the ventrolateral medulla (RVL), is relayed via excitatory synapses to RAm (ex) neurons that make glutamatergic synapses on XIIts MNs and to RAm bulbospinal (BS) neurons that project to the expiratory motor neurons (exp MNs). During song playback/singing, RA appropriates control of brainstem circuits for both respiration and syringeal function. Neurons in ventral RA make excitatory (glutamatergic) synapses on XIIts MNs, whereas dorsal RA neurons make as yet functionally uncharacterized synapses on RVL and on a population of RAm (in) neurons that form inhibitory [glycinergic ( $g l y)$ ] synapses on XIIts MNs. These inhibitory inputs from RAm to XIIts delay XIIts MN activity sufficiently to allow formation of the pressure head generated by the expiratory MNs, thus facilitating syringeal (vocal) and respiratory integration. Numbers (1-3) suggest the sequential timing of the different components of the respiratory-vocal control circuit; phase delays between the respiratory versus vocal muscle groups also could be established within RA (i.e., dorsal vs ventral RA neurons) as well as via other synaptic delays within the brainstem circuitry.

by a phasic membrane hyperpolarization. This behavior could arise through feedforward inhibition driven by RA through the lateral medulla, because RA projection neurons are excitatory (Perkel, 1995) and RA innervates RAm, which, as we show here, provides inhibitory input to XIIts (Wild, 1993b). One idea is that RA selectively recruits otherwise inactive inhibitory inputs to XIIts (Fig. 15), perhaps further enhancing the ability of RA to control the precision of XIIts MN spike timing during singing (Mainen and Sejnowski, 1995). A second idea is that feedforward inhibition to XIIts enables RA to achieve appropriate phase delays between respiratory and vocal activity during singing. That is, RA could directly excite expiratory premotor neurons while indirectly inhibiting XIIts MNs, thereby regulating the relative timing of syringeal and abdominal muscle activity.

In addition to coordinating respiratory and vocal activities, inhibition from ipsilateral and contralateral respiratory areas also may facilitate coordination of the two syringeal halves. One aspect of the song control system, especially in the zebra finch, is that projections of RA to the brainstem are primarily ipsilateral (Wild, 1993b; Wild et al., 2000). These ipsilateral projections contrast markedly with the exquisitely precise bilateral interactions, including lateralized functional asymmetries, that the syrinx exhibits during singing (Suthers, 2000). Although brainstemto-telencephalon feedback could participate in such bilateral interactions (Striedter and Vu, 1998), the speed and intricacy of this coordination may require more direct mechanisms. One of

these probably involves the inhibitory and partly concealed excitatory bilateral projections from RAm to XIIts that we identified here, consistent with previous anatomical evidence of crossed RAm projections (Wild et al., 2000). Another possible source of a crossed influence on XIIts, however, is the contralateral XIIts, given that electrical stimulation of the hypoglossal root could evoke PSPs in both contralateral and ipsilateral XIIts MNs. The anatomical substrate for this is unclear, because intracellular fills of XIIts MNs do not reveal axon collaterals, and unilateral tracer injections into XIIts do not retrogradely label contralateral XIIts MNs. However, tracer injections in XIIts retrogradely label neurons lateral to XIIts (i.e., in medial RAm) but with processes extending into it and axons terminating in the contralateral XIIts (Wild et al., 2000). Stimulating the XIIts root could evoke PSPs in contralateral XIIts, if some XIIts MN axon collaterals contact these RAm neurons immediately lateral to XIIts. Ultimately, either half of the telencephalon could act through crossed projections arising from RAm (and possibly XIIts) to bilaterally coordinate respiratory and vocal activity.

Although the syrinx is uniquely avian, the mechanics of its sound generation resemble those of the vocal folds, and XIIts MNs, like mammalian laryngeal MNs, form the output of a respiratory-vocal control network resembling that in mammals, including possible inhibitory RAm inputs (Holstege, 1989; Goller and Larsen, 1997; Shiba et al., 1997; Yajima and Hayashi, 1997). In turn, RAm in both songbirds and mammals receives projections from higher centers via the midbrain, but the direct projections from RA to RAm and XIIts endow the songbird with a degree of telencephalic control of learned vocal communication perhaps rivaled only by humans (Wild, 1997; VanderHorst et al., 2000; Jürgens, 2002).

\section{References}

Dave A, Margoliash D (2000) Song replay during sleep and computational rules for sensorimotor vocal learning. Science 290:812-816.

Doupe A, Kuhl P (1999) Birdsong and human speech: common themes and mechanisms. Annu Rev Neurosci 22:567-631.

Doupe AJ, Konishi M (1991) Song-selective auditory circuits in the vocal control system of the zebra finch. Proc Natl Acad Sci USA 88:11339-11343.

Dubbeldam J, Bout R (1990) The identification of the motor nuclei innervating the tongue muscles in the mallard (Anas platyrhyncos); an HRP study. Neurosci Lett 119:223-237.

Goller F, Larsen ON (1997) A new mechanism of sound generation in songbirds. Proc Natl Acad Sci USA 94:14787-14791.

Goller F, Suthers R (1996a) Role of syringeal muscles in gating airflow and sound production in singing brown thrashers. J Neurophysiol 75:867-876.

Goller F, Suthers R (1996b) Role of syringeal muscles in controlling the phonology of bird song. J Neurophysiol 76:287-300.

Green D, Swets J (1966) Signal detection theory and psychophysics. New York: Wiley.

Hartley RS, Suthers RA (1990) Lateralization of syringeal function during song production in the canary. J Neurobiol 21:1236-1248.

Holstege G (1989) Anatomical study of the final common path for vocalization in the cat. J Comp Neurol 284:242-252.

Jagadeesh B, Wheat HS, Kontsevich LL, Tyler CW, Ferster D (1997) Direction selectivity of synaptic potentials in simple cells of the cat visual cortex. J Neurophysiol 78:2772-2789.

Janata P, Margoliash D (1999) Gradual emergence of song selectivity in sensorimotor structures of the male zebra finch song system. J Neurosci 19:5108-5118.

Jürgens U (2002) Neural pathways underlying vocal control. Neurosci Biobehav Rev 26:235-225.

Kittelberger J, Mooney R (1999) Lesions of an avian forebrain nucleus that disrupt song development alter synaptic connectivity and transmission in the vocal premotor pathway. J Neurosci 19:9385-9398.

Livingston FS, Mooney R (1997) Development of intrinsic and synaptic 
properties in a forebrain nucleus essential to avian song learning. J Neurosci 17:8997-9009.

Mainen ZF, Sejnowski TJ (1995) Reliability of spike timing in neocortical neurons. Science 268:1503-1506.

Manogue KR, Nottebohm F (1982) Relation of medullary motor nuclei to nerves supplying the vocal tract of the budgerigar (Melopsittacus undulatus). J Comp Neurol 204:384-391.

Manogue KR, Paton, JA (1982) Respiratory gating of activity in the avian vocal control system. Brain Res 247:383-387

Margoliash D, Konishi M (1985) Auditory representation of autogenous song in the song system of white-crowned sparrows. Proc Natl Acad Sci USA 82:5997-6000.

Mooney R (1992) Synaptic basis for developmental plasticity in a birdsong nucleus. J Neurosci 12:2464-2477.

Mooney R (2000) Different subthreshold mechanisms underlie songselectivity in identified HVc neurons of the zebra finch. J Neurosci 20:5420-5436.

Nottebohm F (1968) Auditory experience and song development in the chaffinch (Fringilla coelobs). Ibis 110:549-568.

Nottebohm F, Stokes TM, Leonard CM (1976) Central control of song in the canary, Serinus canarius. J Comp Neurol 165:457-486.

Perkel DJ (1995) Effects of neuromodulators on excitatory synaptic transmission in nucleus RA of the zebra finch. Soc Neurosci Abstr 21:960.

Reinke H, Wild JM (1998) Identification and connections of inspiratory premotor neurons in songbirds and budgerigar. J Comp Neurol 391:147-163.

Rosen M, Mooney R (2000) Intrinsic and extrinsic contributions to auditory selectivity in a song nucleus critical for vocal plasticity. J Neurosci 20:5437-5448.

Shiba K, Satoh I, Kobayashi N, Hayashi F (1996) Multifunctional laryngeal motoneurons: an intracellular study in the cat. J Neurosci 19:2717-2727.

Shiba K, Umezaki T, Zheng Y, Miller AD (1997) The nucleus retroambigualis controls laryngeal muscle activity during vocalizaton in the cat. Exp Brain Res 115:513-519.

Solis MM, Doupe AJ (1997) Anterior forebrain neurons develop selectivity by an intermediate stage of birdsong learning. J Neurosci 17:6447-6462.

Stokes TM, Leonard CM, Nottebohm F (1974) The telencephalon, diencephalon, and mesencephalon of the canary, Serinus canaria, in stereotaxic coordinates. J Comp Neurol 156:337-374.

Striedter GF, Vu ET (1998) Bilateral feedback projections to the forebrain in the premotor network for singing in zebra finches. J Neurobiol 34:27-40.

Suthers R (2000) The motor basis of vocal performance in songbirds. In: The design of animal communication (Hauser M, Konishi M, eds), pp 37-62. Cambridge, MA: MIT.

Theunissen FE, Doupe AJ (1998) Temporal and spectral sensitivity of com- plex auditory neurons in the nucleus HVc of male zebra finches. J Neurosci 18:3786-3802.

VanderHorst GJM, Terasawa E, Ralston III HJ, Holstege G (2000) Monosynaptic projections from the lateral periaqueductal gray to the nucleus retroambiguus in the rhesus monkey: implications for vocalization and reproductive behavior. J Comp Neurol 424:251-268.

Vicario DS (1991a) Contributions of syringeal muscles to respiration and vocalization in the zebra finch. J Neurobiol 22:63-73.

Vicario DS (1991b) Organization of the zebra finch song control system: II. Functional organization of outputs from nucleus Robustus archistriatalis. J Comp Neurol 309:486-494.

Vicario DS (1993) A new brain stem pathway for vocal control in the zebra finch song system. NeuroReport 4:983-986.

Vicario DS, Nottebohm F (1988) Organization of the zebra finch song control system: I. Representation of syringeal muscles in the hypoglossal nucleus. J Comp Neurol 271:346-354.

Vicario DS, Yohay KH (1993) Song-selective auditory input to a forebrain vocal control nucleus in the zebra finch. J Neurobiol 24:488-505.

Wild JM (1993a) The avian nucleus retroambigualis: a nucleus for breathing, singing and calling. Brain Res 606:319-324.

Wild JM (1993b) Descending projections of the songbird nucleus robustus archistriatalis. J Comp Neurol 338:225-241.

Wild JM (1994) The auditory-vocal-respiratory axis in birds. Brain Behav Evol 44:192-209.

Wild JM (1997) Neural pathways for the control of birdsong production. J Neurobiol 33:653-670.

Wild JM, Zeigler HP (1980) Central representation and somatotopic organization of the jaw muscles within the facial and trigeminal nuclei of the pigeon (Columba livia). J Comp Neurol 192:175-201.

Wild JM, Arends J, Zeigler H (1990) Projections of the parabrachial nucleus in the pigeon. J Comp Neurol 293:499-523.

Wild JM, Li D, Eagleton C (1997) Projections of the dorsomedial nucleus of the intercollicular complex (DM) in relation to respiratory-vocal nuclei in the brainstem of pigeon (Columba livia) and zebra finch (Taeniopygia guttata). J Comp Neurol 377:392-413.

Wild JM, Williams M, Suthers R (2000) Neural pathways for bilateral vocal control in songbirds. J Comp Neurol 423:413-426.

Williams H, Nottebohm F (1985) Auditory responses in avian vocal motor neurons: a motor theory for song perception in birds. Science 229:279-282.

Yajima Y, Hayashi Y (1997) GABA(A) receptor-mediated inhibition in the nucleus ambiguus motoneuron. Neuroscience 79:1079-1088.

Yu AC, Margoliash D (1996) Temporal hierarchical control of singing in birds. Science 273:1871-1875. 